\title{
A New Kind of Fuzzy $n$-ary Hypergroups in the Framework of Soft Set Theory
}

\author{
Hongjie $\mathrm{Li}^{1}$ and Yunqiang Yin ${ }^{2}$ \\ ${ }^{1}$ Mathematics Department of Zhoukou Normal University, Zhoukou 466001, China \\ ${ }^{2}$ School of Sciences, East China Institute of Technology, Nanchang 330013, China \\ Correspondence should be addressed to Yunqiang Yin; yunqiangyin@gmail.com
}

Received 3 February 2014; Accepted 27 February 2014; Published 14 July 2014

Academic Editor: Jianming Zhan

Copyright (C) 2014 H. Li and Y. Yin. This is an open access article distributed under the Creative Commons Attribution License, which permits unrestricted use, distribution, and reproduction in any medium, provided the original work is properly cited.

\begin{abstract}
Maji et al. introduced the concept of fuzzy soft sets as a generalization of the standard soft sets and presented an application of fuzzy soft sets in a decision making problem. The aim of this paper is to apply the concept of fuzzy soft sets to $n$-ary hypergroup theory. The concepts of $\left(\epsilon_{\gamma}, \epsilon_{\gamma} \vee q_{\delta}\right)$-fuzzy soft (invertible) $n$-ary subhypergroups over a commutative $n$-ary hypergroup are introduced and some related properties and characterizations are obtained. The homomorphism properties of $\left(\epsilon_{\gamma}, \epsilon_{\gamma} \vee q_{\delta}\right)$-fuzzy soft (invertible) $n$-ary subhypergroups are also derived.
\end{abstract}

\section{Introduction}

To solve complicated problems in economics, engineering, and environment, we cannot successfully use classical methods because of various uncertainties typical for those problems. There are three theories: theory of probability, theory of fuzzy sets, and the interval mathematics, which we can consider as mathematical tools for dealing with uncertainties. However, all of these have their advantages as well as inherent limitations in dealing with uncertainties. One major problem shared by those theories is their incompatibility with the parameterization tools. To overcome these difficulties, Molodtsov [1] introduced the concept of soft set as a new mathematical tool for dealing with uncertainties that is free from the difficulties that have troubled the usual theoretical approaches. Molodtsov pointed out several directions for the applications of soft sets. This theory has proven useful in many different fields such as decision making [2-6], data analysis [7, 8], and forecasting [9].

Up to the present, research on soft sets has been very active and many important results have been achieved in the theoretical aspect. Maji et al. [10] introduced several algebraic operations in soft set theory and published a detailed theoretical study on soft sets. Ali et al. [11] further presented and investigated some new algebraic operations for soft sets. Qin and Hong [12] further investigated the algebraic structure of soft sets and established the soft quotient algebra. Maji et al. [13] and Majumdar and Samanta [14] extended (classical) soft sets to fuzzy soft sets, respectively. Maji et al. [15] extended (classical) soft sets to intuitionistic fuzzy soft sets, which were further discussed by Maji et al. [16] and Jiang et al. [17]. Aktaş and Çağman [18] compared soft sets to the related concepts of fuzzy sets and rough sets. They also defined the notion of soft groups and derived some related properties. Aygünoğlu and Aygün [19] discussed the applications of fuzzy soft sets to group theory and investigated (normal) fuzzy soft groups. Feng et al. [20] investigated soft semirings by using the soft set theory. Jun [21] introduced and investigated the notion of soft BCK/BCI-algebras. Jun and Park [22] and Jun et al. [23] discussed the applications of soft sets in ideal theory of BCK/BCI-algebras and in $d$ algebras, respectively. Acar et al. [24] introduced and studied soft rings. Zhan and Jun [25] characterized the (implicative, positive implicative, and fantastic) filteristic soft $B L$-algebras based on $\epsilon$-soft sets and $q$-soft sets.

On the other hand, the fuzzy sets and hyperstructures introduced by Zadeh and Marty, respectively, are now extensively applied to many disciplines. The study of fuzzy hyperstructures is an interesting research topic of fuzzy sets. There is a considerable amount of work on the connections between 
fuzzy sets and hyperstructures. The reader is referred to [2638]. As a generalization of the concepts of "belongingness $(\epsilon)$ " and "quasi-coincidence $(q)$ " of a fuzzy point with a fuzzy set introduced by $\mathrm{Pu}$ and Liu [39], Yin and Zhan [40] introduced the concepts of " $\gamma$-belongingness $\left(\epsilon_{\gamma}\right)$ " and " $\delta$ quasi-coincidence $\left(q_{\delta}\right)$ " of a fuzzy point with a fuzzy set. Using these new concepts, Yin and Zhan [40] introduced the concepts of $(\alpha, \beta)$-fuzzy (implicative, positive implicative, and fantastic) filters and $(\bar{\beta}, \bar{\alpha})$-fuzzy (implicative, positive implicative, and fantastic) filters of $B L$-algebras, where $\alpha, \beta \epsilon$ $\left\{\epsilon_{\gamma}, q_{\delta}, \epsilon_{\gamma} \wedge q_{\delta}, \epsilon_{\gamma} \vee q_{\delta}\right\}, \bar{\alpha}, \bar{\beta} \in\left\{\overline{\epsilon_{\gamma}}, \overline{q_{\delta}}, \overline{\epsilon_{\gamma}} \wedge \overline{q_{\delta}}, \overline{\epsilon_{\gamma}} \vee \overline{q_{\delta}}\right\}$, $\alpha \neq \epsilon_{\gamma} \wedge q_{\delta}$, and $\bar{\beta} \neq \overline{\epsilon_{\gamma}} \wedge \overline{q_{\delta}}$, and some related properties were investigated. Special attention is paid to $\left(\overline{\epsilon_{\gamma}}, \overline{\epsilon_{\gamma}} \vee \overline{q_{\delta}}\right)$ fuzzy (implicative, positive implicative, and fantastic) filters and $\left(\overline{\epsilon_{\gamma}}, \overline{\epsilon_{\gamma}} \vee \overline{q_{\delta}}\right)$-fuzzy (implicative, positive implicative, and fantastic) filters which are generalizations of $(\epsilon, \in \vee q)$ fuzzy (implicative, positive implicative, and fantastic) filters and $(\bar{\epsilon}, \bar{\epsilon} \vee \bar{q}$ )-fuzzy (implicative, positive implicative, and fantastic) filters studied by Ma et al. [41, 42]. This idea is continued and studied by Ma et al. [43], Yin and Zhan [44, 45], Yin et al. [46], and so on. The purpose of this paper is to deal with the algebraic structure of $n$-ary hypergroups by applying fuzzy soft set theory. We introduce the concepts of $\left(\epsilon_{\gamma}, \epsilon_{\gamma} \vee q_{\delta}\right)$-fuzzy soft (invertible) $n$-ary subhypergroups over a commutative $n$-ary hypergroup and focus on investigating their characterization and algebraic properties. We also discuss the homomorphism properties of $\left(\epsilon_{\gamma}, \epsilon_{\gamma} \vee q_{\delta}\right)$-fuzzy soft (invertible) $n$-ary subhypergroups.

\section{Preliminaries}

In this section, we recall some basic notions and results about $n$-ary hypergroups, fuzzy sets, and fuzzy soft sets (see $[13,28$, $30,47])$.

2.1. $n$-Ary Hypergroups. We will be concerned primarily with a basic nonempty set $H$. Denote by $H^{n}$ the cartesian product $H \times \cdots \times H$, where $H$ appears $n$ times. An element of $H^{n}$ will be denoted by $\left(x_{1}, \ldots, x_{n}\right)$, where $x_{i} \in H$ for all $1 \leq i \leq n$. In general, a mapping $f: H^{n} \rightarrow \mathscr{P}^{*}(H)$ is called an $n$-ary hyperoperation and $n$ is called the arity of the hyperoperation $f$. Let $f$ be an $n$-ary hyperoperation on $H$ and $A_{1}, \ldots, A_{n}$ subsets in $H$. Define

$$
f\left(A_{1}, \ldots, A_{n}\right)=\cup\left\{f\left(x_{1}, \ldots, x_{n}\right) \mid x_{i} \in A_{i}, 1 \leq i \leq n\right\} .
$$

In the sequel, we will denote the sequence $x_{i}, x_{i+1}, \ldots, x_{j}$ by $x_{i}^{j}$. For $j<i, x_{i}^{j}=\emptyset$. Thus,

$$
f\left(x_{1}, \ldots, x_{i}, y_{i+1}, \ldots, y_{j}, z_{j+1}, \ldots, z_{n}\right)
$$

will be written as $f\left(x_{1}^{i}, y_{i+1}^{j}, z_{j+1}^{n}\right)$. Also $f\left(a_{1}^{i}, x *\right)$ means $f(a_{1}^{i}, \underbrace{x, \ldots, x}_{n-i})$ for $a_{1}^{i}, x \in H$ and $1 \leq i \leq n$.

$H$ with an $n$-ary hyperoperation $f: H^{n} \rightarrow \mathscr{P}^{*}(H)$ is called an n-ary hypergroupoid and will be denoted by
$(H, f)$. An $n$-ary hypergroupoid $(H, f)$ is said to be an $n$-ary semihypergroup if the following associative axiom holds:

$$
f\left(x_{1}^{i-1}, f\left(x_{i}^{n+i-1}\right), x_{n+i}^{2 n-1}\right)=f\left(x_{1}^{j-1}, f\left(x_{j}^{n+j-1}\right), x_{n+j}^{2 n-1}\right)
$$

for all $i, j \in\{1, \ldots, n\}$ and $x_{1}^{2 n-1} \in H$. An $n$-ary semihypergroup is said to be an n-ary hypergroup if, for all $y, y_{1}^{n} \epsilon$ $H$ and fixed $i \in\{1, \ldots, n\}$, there exists $x \in H$ such that $y \in f\left(y_{1}^{i-1}, x, y_{i+1}^{n}\right)$. An $n$-ary hypergroup is said to be commutative if, for all $x_{1}^{n} \in H$ and any permutation $\rho$ of $\{1, \ldots, n\}$, we have $f\left(x_{1}^{n}\right)=f\left(x_{\rho_{1}}, \ldots, x_{\rho_{n}}\right)$. An element $e$ of $H$ is called an identity of $H$ if, for any $x \in H$ and $i \in\{1, \ldots, n\}$, we have $x \in f(\underbrace{e, \ldots, e}_{i-1}, x, \underbrace{e, \ldots, e}_{n-i})$.

Definition 1. Let $(H, f)$ be an $n$-ary hypergroup and $K$ a nonempty subset of $H$. One says that $K$ is an $n$-ary subhypergroup of $H$ if the following conditions hold:

(i) $K$ is closed under the $n$-ary hyperoperation $f$;

(ii) for all $y, y_{1}^{n} \in K$ and fixed $i \in\{1, \ldots, n\}$, there exists $x \in K$ such that $y \in f\left(y_{1}^{i-1}, x, y_{i+1}^{n}\right)$.

In what follows, $(H, f)$ denotes a commutative $n$-ary hypergroup with an identity $e$ unless otherwise stated.

An $n$-ary subhypergroup $K$ of $H$ is said to be invertible if, for any $x, y \in H, x \in f(y, \underbrace{K, \ldots, K}_{n-1})$ implies $y \in$ $f(x, \underbrace{K, \ldots, K}_{n-1})$.

2.2. Fuzzy Sets. Let $X$ be a nonempty set. A fuzzy subset $\mu$ of $X$ is defined as a mapping from $X$ into $[0,1]$, where $[0,1]$ is the usual interval of real numbers. We denote by $\mathscr{F}(X)$ the set of all fuzzy subsets of $X$. For $\mu, v \in \mathscr{F}(X)$, by $\mu \subseteq \nu$, we mean $\mu(x) \leq \nu(x)$ for all $x \in X$. And the union and intersection of $\mu$ and $\nu$, denoted by $\mu \cup \nu$ and $\mu \cap \nu$, are defined as the fuzzy subsets of $X$ by $(\mu \cup \nu)(x)=\max \{\mu(x), \nu(x)\}$ and $(\mu \cap \nu)(x)=$ $\min \{\mu(x), \nu(x)\}$ for all $x \in X$.

For $x_{i}, x_{i+1}, \ldots, x_{j} \in X$ and $\mu \in \mathscr{F}(X)$, the sequence $\mu\left(x_{i}\right), \mu\left(x_{i+1}\right), \ldots, \mu\left(x_{j}\right)$ is denoted by $\mu_{x_{i}}^{x_{j}}$, and $\mu_{x_{i}}^{x_{j}}=0$ if $j<i$. Moreover, for $\mu_{i}, \mu_{i+1}, \ldots, \mu_{j} \in \mathscr{F}(X)$, the sequence $\mu_{i}, \mu_{i+1}, \ldots, \mu_{j}$ is denoted by $\mu_{i}^{j}$, and $\mu_{i}^{j}$ denotes the zero fuzzy set if $j<i$. $X$ by

For any $Y \subseteq X$ and $r \in(0,1]$, define a fuzzy subset $r_{Y}$ of

$$
r_{Y}(x)=\left\{\begin{array}{lc}
r & \text { if } x \in Y \\
0 & \text { otherwise }
\end{array}\right.
$$

for all $x \in X$. In particular, when $r=1, r_{Y}$ is said to be the characteristic function of $Y$, denoted by $\chi_{Y}$, and when $X=$ $\{x\}, r_{X}$ is said to be a fuzzy point with support $x$ and value $r$ and is denoted by $r_{x}$.

In what follows, let $\gamma, \delta \in[0,1]$ be such that $\gamma<\delta$. For a fuzzy point $r_{x}$ and a fuzzy subset $\mu$ of $X$, we say that

(1) $r_{x} \in_{\gamma} \mu$ if $\mu(x) \geq r>\gamma$; 
(2) $r_{x} q_{\delta} \mu$ if $\mu(x)+r>2 \delta$;

(3) $r_{x} \epsilon_{\gamma} \vee q_{\delta} \mu$ if $r_{x} \epsilon_{\gamma} \mu$ or $r_{x} q_{\delta} \mu[40]$.

Let us now introduce a new relation on $\mathscr{F}(X)$, denoted by " $\subseteq \vee q_{(\gamma, \delta)}$," as follows.

For any $\mu, \nu \in \mathscr{F}(X)$, by $\mu \subseteq \vee q_{(\gamma, \delta)} \nu$, we mean that $r_{x} \epsilon_{\gamma} \mu$ implies $r_{x} \epsilon_{\gamma} \vee q_{\delta} \nu$ for all $x \in X$ and $r \in(\gamma, 1]$. Moreover, $\mu$ and $\nu$ are said to be $(\gamma, \delta)$-equal, denoted by $\mu={ }_{(\gamma, \delta)} \nu$, if $\mu \subseteq \vee q_{(\gamma, \delta)} \nu$ and $\nu \subseteq \vee q_{(\gamma, \delta)} \mu$.

In the sequel, unless otherwise stated, $\bar{\alpha}$ means that $\alpha$ does not hold, where $\alpha \in\left\{\epsilon_{\gamma}, q_{\delta}, \epsilon_{\gamma} \vee q_{\delta}, \subseteq \vee q_{(\gamma, \delta)}\right\}$.

Lemma 2 (see [40]). Let $\mu, v \in \mathscr{F}(X)$. Then $\mu \subseteq \vee q_{(\gamma, \delta)} \nu$ if and only if $\max \{\nu(x), \gamma\} \geq \min \{\mu(x), \delta\}$ for all $x \in X$.

Lemma 3 (see [40]). Let $\mu, \nu, \omega \in \mathscr{F}(X)$. If $\mu \subseteq \vee q_{(\gamma, \delta)} \nu$ and $\nu \subseteq \vee q_{(\gamma, \delta)} \omega$, then $\mu \subseteq \vee q_{(\gamma, \delta)} \omega$.

Proof. It is straightforward by Lemma 2

Lemmas 2 and 3 give that " $=(\gamma, \delta)$ " is an equivalence relation on $\mathscr{F}(X)$. It is also worth noticing that $\mu=_{(\gamma, \delta)} \nu$ if and only if $\max \{\min \{\mu(x), \delta\}, \gamma\}=\max \{\min \{\nu(x), \delta\}, \gamma\}$ for all $x \in X$ by Lemma 2

2.3. Fuzzy Soft Sets. Let $U$ be an initial universe set and $E$ the set of all possible parameters under consideration with respect to $U$. As a generalization of soft set introduced by Molodtsov [1], Maji et al. [13] defined fuzzy soft set in the following way.

Definition 4. A pair $\langle F, A\rangle$ is called a fuzzy soft set over $U$, where $A \subseteq E$ and $F$ is a mapping given by $F: A \rightarrow \mathscr{F}(U)$.

In general, for every $\varepsilon \in A, F(\varepsilon)$ is a fuzzy set of $U$ and it is called fuzzy value set of parameter $\varepsilon$. The set of all fuzzy soft sets over $U$ with parameters from $E$ is called fuzzy soft classes, and it is denoted by $\mathscr{F} \mathcal{S}(U, E)$. A fuzzy soft set $\langle F, A\rangle$ in $\mathscr{F} \mathcal{S}(U, E)$ is said to have sup-property if, for any $V \in \mathscr{P}^{*}(U)$ and $\varepsilon \in A$, there exists $x_{0} \in V$ such that $F(\varepsilon)\left(x_{0}\right)=\sup _{x \in V} F(\varepsilon)(x)$.

Let $A \subseteq E$ and $\mu \in \mathscr{F}(U)$. Define $\langle\widetilde{\mu}, A\rangle$ to be a fuzzy soft set by $\widetilde{\mu}(\varepsilon)=\mu$ for all $\varepsilon \in A$. For any fuzzy point $r_{x}$ in $U$, define $\left(\widetilde{r_{x}}, A\right)$ to be a fuzzy soft set by $\widetilde{r_{x}}(\varepsilon)=r_{x}$ for all $\varepsilon \in A$.

Definition 5 (see [6]). Let $\langle F, A\rangle$ and $\langle G, B\rangle$ be two fuzzy soft sets over $U$. One says that $\langle F, A\rangle$ is a fuzzy soft subset of $\langle G, B\rangle$ and writes $\langle F, A\rangle \Subset\langle G, B\rangle$ if

(i) $A \subseteq B$;

(ii) for any $\varepsilon \in A, F(\varepsilon) \subseteq G(\varepsilon)$.

$\langle F, A\rangle$ and $\langle G, B\rangle$ are said to be fuzzy soft equal and write $\langle F, A\rangle=\langle G, B\rangle$ if $\langle F, A\rangle \Subset\langle G, B\rangle$ and $\langle G, B\rangle \Subset\langle F, A\rangle$.

Let us now introduce some new concepts on fuzzy soft sets analogous to the concepts introduced by Ali et al. [11].
Definition 6. The extended intersection of two fuzzy soft sets $\langle F, A\rangle$ and $\langle G, B\rangle$ over $U$ is a fuzzy soft set denoted by $\langle H, C\rangle$, where $C=A \cup B$ and

$$
H(\varepsilon)= \begin{cases}F(\varepsilon) & \text { if } \varepsilon \in A-B \\ G(\varepsilon) & \text { if } \varepsilon \in B-A \\ F(\varepsilon) \cap G(\varepsilon) & \text { if } \varepsilon \in A \cap B\end{cases}
$$

for all $\varepsilon \in C$. This is denoted by $\langle H, C\rangle=\langle F, A\rangle \widetilde{\cap}\langle G, B\rangle$.

Definition 7. Let $\langle F, A\rangle$ and $\langle G, B\rangle$ be two fuzzy soft sets over $U$ such that $A \cap B \neq \emptyset$. The restricted intersection of $\langle F, A\rangle$ and $\langle G, B\rangle$ is defined to be the fuzzy soft set $\langle H, C\rangle$, where $C=$ $A \cap B$ and $H(\varepsilon)=F(\varepsilon) \cap G(\varepsilon)$ for all $\varepsilon \in C$. This is denoted by $\langle H, C\rangle=\langle F, A\rangle \cap\langle G, B\rangle$.

Definition 8. Let $V \subseteq U$ and $r \in(\gamma, 1]$. A fuzzy soft set $\langle F, A\rangle$ over $V$ is said to be a relative whole $(\gamma, \delta)$-fuzzy soft set (with respect to universe set $V$, parameter set $A$, and value $r$ ), denoted by $\Sigma(V, A, r)$, if $F(\varepsilon)=r_{V}$ for all $\varepsilon \in A$.

Definition 9. Let $\langle F, A\rangle$ and $\langle G, B\rangle$ be two fuzzy soft sets over $U$. One says that $\langle F, A\rangle$ is $(\gamma, \delta)$-fuzzy soft subset of $\langle G, B\rangle$ and writes $\langle F, A\rangle \Subset_{(\gamma, \delta)}\langle G, B\rangle$ if

(i) $A \subseteq B$;

(ii) for any $\varepsilon \in A, F(\varepsilon) \subseteq \vee q_{(\gamma, \delta)} G(\varepsilon)$.

$\langle F, A\rangle$ and $\langle G, B\rangle$ are said to be $(\gamma, \delta)$-fuzzy soft equal and write $\langle F, A\rangle \asymp_{(\gamma, \delta)}\langle G, B\rangle$ if $\langle F, A\rangle \Subset_{(\gamma, \delta)}\langle G, B\rangle$ and $\langle G, B\rangle \Subset_{(\gamma, \delta)}\langle F, A\rangle$.

Clearly, $\langle F, A\rangle \Subset\langle G, B\rangle$ implies $\langle F, A\rangle \subseteq \vee q_{(\gamma, \delta)}\langle G, B\rangle$ by Lemma 2 and Definition 9.

Lemma 10. Let $\langle F, A\rangle,\langle G, B\rangle$, and $\langle H, C\rangle$ be fuzzy soft sets over $U$. If $\langle F, A\rangle \Subset_{(\gamma, \delta)}\langle G, B\rangle$ and $\langle G, B\rangle \Subset_{(\gamma, \delta)}\langle H, C\rangle$, then $\langle F, A\rangle \Subset_{(\gamma, \delta)}\langle F, C\rangle$.

Proof. It is straightforward by Lemma 3 and Definition 9.

Lemmas 2 and 10 and Definition 9 give that " $\asymp_{(\gamma, \delta)}$ ” is an equivalence relation on $\mathscr{F} \mathcal{S}(U, E)$.

\section{A New Operation on Fuzzy Soft Sets over an n-Ary Hypergroupoid}

We first introduce a fuzzy $n$-ary hyperoperation on an $n$-ary hypergroupoid as follows.

Definition 11. Let $(H, f)$ be an $n$-ary hypergroupoid and $\mu_{1}, \ldots, \mu_{n} \in \mathscr{F}(H)$. Define a fuzzy $n$-ary hyperoperation $\mathscr{F}: \underbrace{\mathscr{F}(H) \times \cdots \times \mathscr{F}(H)}_{n} \rightarrow \mathscr{F}(H)$ by

$$
\mathscr{F}\left(\mu_{1}, \ldots, \mu_{n}\right)(x)=\sup _{x \in f\left(y_{1}^{n}\right)} \min \left\{\mu_{1}\left(y_{1}\right), \ldots, \mu_{n}\left(y_{n}\right)\right\}
$$


for all $x \in H$. In particular, for any $x_{1}^{n} \in H$ and $i \in\{1, \ldots, n\}$, define

$$
\begin{aligned}
\mathscr{F} & \left(\mu_{1}, \ldots, \mu_{i}, x_{i+1}^{n}\right)(x) \\
& =\sup _{x \in f\left(y_{1}^{i}, x_{i+1}^{n}\right)} \min \left\{\mu_{1}\left(y_{1}\right), \ldots, \mu_{i}\left(y_{i}\right)\right\}
\end{aligned}
$$

for all $x \in H$.

Clearly, for any $x_{1}^{n} \in H$ and $1 \leq i \leq n$, $\mathscr{F}\left(\mu_{1}, \ldots, \mu_{i}, x_{i+1}^{n}\right)=\mathscr{F}\left(\mu_{1}, \ldots, \mu_{i}, 1_{x_{i+1}}, \ldots, 1_{x_{n}}\right)$. Note that, for any $r_{1}^{n} \in(0,1]$ and $x_{1}^{n} \in H$, by Definition 11, $\mathscr{F}\left(r_{1_{x_{1}}}, r_{2_{x_{2}}}, \ldots, r_{n_{x_{n}}}\right)=\min \left\{r_{1}^{n}\right\}_{f\left(x_{1}^{n}\right)}$

Definition 12. Let $(H, f)$ be an $n$-ary hypergroupoid and $\left\langle F_{i}, A_{i}\right\rangle \in \mathscr{F} \mathcal{S}(H, E)$, where $i \in I$ and $I$ is an index set, such that $\bigcap_{i \in I} A_{i} \neq \emptyset$. One defines a fuzzy soft set over $H$, denoted by $\left\langle\mathbb{F}\left(F_{1}, \ldots, F_{n}\right), C\right\rangle$, where $C=\bigcap_{i \in I} A_{i}$ and

$$
\mathbb{F}\left(F_{1}, \ldots, F_{n}\right)(\varepsilon)=\mathscr{F}\left(F_{1}(\varepsilon), \ldots, F_{n}(\varepsilon)\right),
$$

for all $\varepsilon \in C$. This is denoted by $\left\langle\mathbb{F}\left(F_{1}, \ldots, F_{n}\right), C\right\rangle=$ $\mathfrak{F}\left(\left\langle F_{1}, A_{1}\right\rangle, \ldots,\left\langle F_{n}, A_{n}\right\rangle\right)$.

In the following, we will denote the sequence $\left\langle F_{i}, A_{i}\right\rangle,\left\langle F_{i+1}, A_{i+1}\right\rangle, \ldots,\left\langle F_{j}, A_{j}\right\rangle$ by $\langle F, A\rangle_{i}^{j}$. For $j<i$, $\langle F, A\rangle_{i}^{j}$ is the empty set. The following elementary facts follow easily from the definition.

Lemma 13. Let $(H, f)$ be an n-ary hypergroupoid and $\langle F, A\rangle_{1}^{2 n-1},\langle G, B\rangle_{1}^{n} \in \mathscr{F} \mathcal{S}(H, E)$. Then

(1) if $(H, f)$ is an $n$-ary semihypergroup and $\bigcap_{i=1}^{2 n} A_{i} \neq \emptyset$, then

$$
\begin{aligned}
\mathfrak{F} & \left(\langle F, A\rangle_{1}^{i-1}, \mathfrak{F}\left(\langle F, A\rangle_{i}^{n+i-1}\right),\langle F, A\rangle_{n+i}^{2 n-1}\right) \\
& =\mathfrak{F}\left(\langle F, A\rangle_{1}^{j-1}, \mathfrak{F}\left(\langle F, A\rangle_{j}^{n+j-1}\right),\langle F, A\rangle_{n+j}^{2 n-1}\right)
\end{aligned}
$$

for all $1 \leq i, j \leq n$;

(2) if $(H, f)$ is commutative and $\bigcap_{i=1}^{n} A_{i} \neq \emptyset$, then the value of $\mathfrak{F}\left(\langle F, A\rangle_{1}^{n}\right)$ does not depend on the permutation of $\left\langle F_{1}, A_{1}\right\rangle, \ldots,\left\langle F_{n}, A_{n}\right\rangle$;

(3) if $\left\langle F_{i}, A_{i}\right\rangle \Subset_{(\gamma, \delta)}\left\langle G_{i}, B_{i}\right\rangle$ for all $1 \leq i \leq n$ and $\bigcap_{i=1}^{n} A_{i} \neq \emptyset$, then $\mathfrak{F}\left(\langle F, A\rangle_{1}^{n}\right) \epsilon_{(\gamma, \delta)} \mathfrak{F}\left(\langle G, B\rangle_{1}^{n}\right)$;

(4) if $\left\langle F_{1}, A_{1}\right\rangle \Subset_{(\gamma, \delta)}\left\langle G_{1}, A_{1}\right\rangle$ and $\left\langle F_{2}, A_{2}\right\rangle \Subset_{(\gamma, \delta)}\left\langle G_{2}, A_{2}\right\rangle$, then $\left\langle F_{1}, A_{1}\right\rangle \tilde{\cap}\left\langle F_{2}, A_{2}\right\rangle \Subset_{(\gamma, \delta)}\left\langle G_{1}, A_{1}\right\rangle \widetilde{\cap}\left\langle G_{2}, A_{2}\right\rangle$ and $\left\langle F_{1}, A_{1}\right\rangle$ ก $\left\langle F_{2}, A_{2}\right\rangle \Subset_{(\gamma, \delta)}\left\langle G_{1}, A_{1}\right\rangle$ ก $\left\langle G_{2}, A_{2}\right\rangle$ if $A_{1} \cap$ $A_{2} \neq \emptyset$.

\section{4. $\left(\epsilon_{\gamma}, \epsilon_{\gamma} \vee q_{\delta}\right)$-Fuzzy Soft (Invertible) n-Ary Subhypergroups over a Commutative $n$-Ary Hypergroup}

In this section, we will introduce and investigate the concept of $\left(\epsilon_{\gamma}, \epsilon_{\gamma} \vee q_{\delta}\right)$-fuzzy soft $n$-ary subhypergroups over a commutative $n$-ary hypergroup. Let us start by giving the following concept.
Definition 14. Let $\langle F, A\rangle \in \mathscr{F} \mathcal{S}(H, E)$. Then $\langle F, A\rangle$ is called $\left(\epsilon_{\gamma}, \epsilon_{\gamma} \vee q_{\delta}\right)$-fuzzy soft n-ary subhypergroup over $H$ if it satisfies the following conditions:

(F1a) for all $x \in H$ and $\varepsilon \in A, \max \{F(\varepsilon)(e), \gamma\} \geq$ $\min \{F(\varepsilon)(x), \delta\}$

(F2a) for all $x_{1}^{n} \in H$ and $\varepsilon \in A, \max \left\{\inf _{x \in f\left(x_{1}^{n}\right)} F(\varepsilon)(x), \gamma\right\} \geq$ $\min \left\{F(\varepsilon)_{x_{1}}^{x_{n}}, \delta\right\}$

(F3a) for all $y, y_{1}^{n-1} \in H$ and $\varepsilon \in A$, there exists $x \in H$ such that

$$
y \in f\left(x, y_{1}^{n-1}\right)
$$

$$
\max \{F(\varepsilon)(x), \gamma\} \geq \min \left\{F(\varepsilon)_{y_{1}}^{y_{n-1}}, F(\varepsilon)(y), \delta\right\} .
$$

An $\left(\epsilon_{\gamma}, \epsilon_{\gamma} \vee q_{\delta}\right)$-fuzzy $n$-ary subhypergroup $\langle F, A\rangle$ over $H$ is said to be invertible if it satisfies the following:

(F4a) for all $x, y \in H$ and $\varepsilon \in A$,

$$
\begin{aligned}
& \max \{\min \{\mathscr{F}(y, \underbrace{F(\varepsilon), \ldots, F(\varepsilon)}_{n-1})(x), \delta\}, \gamma\} \\
& =\max \{\min \{\mathscr{F}(x, \underbrace{F(\varepsilon), \ldots, F(\varepsilon)}_{n-1})(y), \delta\}, \gamma\} .
\end{aligned}
$$

Example 15. Let $Z_{8}$ be the residue class of $(Z,+)$ modulo 8 with an $n$-ary hyperoperation $f$ as follows:

$$
\begin{gathered}
f: Z_{8}^{n} \longrightarrow \mathscr{P}^{*}\left(Z_{8}\right), \\
f\left(x_{1}^{n}\right)=\left\{m_{1} x_{1}+\cdots+m_{n} x_{n} \mid m_{1}, \ldots, m_{n} \in Z\right\} .
\end{gathered}
$$

Then, $\left(Z_{8}, f\right)$ is a commutative $n$-ary hypergroup. Let $E=$ $(0.2,0.6]$ and $r \geq 0.6$. Define a fuzzy soft set $\langle F, A\rangle$ over $Z_{8}$ by

$$
F(\varepsilon)(x)= \begin{cases}r & \text { if } x=0 \\ \varepsilon & \text { if } x \in\{2,4\}, \\ 0.2 & \text { otherwise }\end{cases}
$$

for all $\varepsilon \in E$ and $x \in Z_{8}$. Then $\langle F, E\rangle$ is an $\left(\epsilon_{0.2}, \epsilon_{0.2} \vee q_{0.6}\right)$ fuzzy soft invertible $n$-ary subhypergroup over $Z_{8}$.

Next let us first provide some auxiliary lemmas as follows.

Lemma 16. Let $\langle F, A\rangle \in \mathscr{F} \mathcal{S}(H, E)$. Then (F2a) holds if and only if one of the following conditions holds:

(F2b) $\mathfrak{F}(\underbrace{\langle F, A\rangle, \ldots,\langle F, A\rangle}_{n}) \Subset_{(\gamma, \delta)}\langle F, A\rangle$;

(F2c) for all $x_{1}^{n} \in H$ and $\varepsilon \quad \in \quad A$, $\mathscr{F}\left(F(\varepsilon)\left(x_{1}\right)_{x_{1}}, \ldots, F(\varepsilon)\left(x_{n}\right)_{x_{n}}\right) \subseteq \vee q_{(\gamma, \delta)} F(\varepsilon) ;$

(F2d) for all $\varepsilon \in A$ and $r_{i_{x_{i}}} \epsilon_{\gamma} F(\varepsilon)(1 \leq i \leq n)$, $\mathscr{F}\left(r_{1_{x_{1}}}, \ldots, r_{n_{x_{n}}}\right) \subseteq \vee q_{(\gamma, \delta)} F(\varepsilon)$. 
Proof. (F2a) $\Rightarrow$ (F2b) Assume that (F2a) holds. Then, for any $x \in H$ and $\varepsilon \in A$, we have

$$
\begin{aligned}
& \min \{\mathscr{F}(\underbrace{F(\varepsilon), \ldots, F(\varepsilon)}_{n})(x), \delta\} \\
& =\min \left\{\sup _{x \in f\left(y_{1}^{n}\right)} \min \left\{F(\varepsilon)_{y_{1}}^{y_{n}}\right\}, \delta\right\} \\
& =\sup _{x \in f\left(y_{1}^{n}\right)} \min \left\{F(\varepsilon)_{y_{1}}^{y_{n}}, \delta\right\} \leq \max \{F(\varepsilon)(x), \gamma\}
\end{aligned}
$$

and so $\mathscr{F}(\underbrace{F(\varepsilon), \ldots, F(\varepsilon)}_{n}) \subseteq \vee q_{(\gamma, \delta)} F(\varepsilon)$ by Lemma 2. Therefore, $\mathfrak{F}(\underbrace{\langle F, A\rangle, \ldots,\langle F, A\rangle}_{n}) \Subset_{(\gamma, \delta)}\langle F, A\rangle$.

$(\mathrm{F} 2 \mathrm{~b}) \Rightarrow(\mathrm{F} 2 \mathrm{c})$ Let $x_{1}^{n} \in H$ and $\varepsilon \in A$. Since $F(\varepsilon)\left(x_{i}\right)_{x_{i}} \in F(\varepsilon)$, for all $1 \leq i \leq n$, we have

$$
\begin{aligned}
\mathscr{F} & \left(F(\varepsilon)\left(x_{1}\right)_{x_{1}}, \ldots, F(\varepsilon)\left(x_{n}\right)_{x_{n}}\right) \\
& \subseteq \mathscr{F}(\underbrace{F(\varepsilon), \ldots, F(\varepsilon)}_{n}) \subseteq \vee q_{(\gamma, \delta)} F(\varepsilon),
\end{aligned}
$$

and so $\mathscr{F}\left(F(\varepsilon)\left(x_{1}\right)_{x_{1}}, \ldots, F(\varepsilon)\left(x_{n}\right)_{x_{n}}\right) \subseteq \vee q_{(\gamma, \delta)} F(\varepsilon)$.

(F2c) $\Leftrightarrow$ (F2d) This is straightforward.

(F2d) $\Rightarrow$ (F2a) Let $\varepsilon \in A, x \in H$, and $x_{1}^{n} \in H$ be such that $x \in f\left(x_{1}^{n}\right)$. Then, by Lemma 2 and (F2d), we have

$$
\begin{aligned}
& \max \{F(\varepsilon)(x), \gamma\} \\
& \geq \min \left\{\mathscr{F}\left(F(\varepsilon)\left(x_{1}\right)_{x_{1}}, \ldots, F(\varepsilon)\left(x_{n}\right)_{x_{n}}\right)(x), \delta\right\} \\
& =\min \left\{\left(\min \left\{F(\varepsilon)_{x_{1}}^{x_{n}}\right\}_{f\left(x_{1}^{n}\right)}\right)(x), \delta\right\} \\
& =\min \left\{F(\varepsilon)_{x_{1}}^{x_{n}}, \delta\right\} .
\end{aligned}
$$

This implies $\max \left\{\inf _{x \in f\left(x_{1}^{n}\right)} F(\varepsilon)(x), \gamma\right\} \geq \min \left\{F(\varepsilon)_{x_{1}}^{x_{n}}, \delta\right\}$ for all $x_{1}^{n} \in H$ and $\varepsilon \in A$. Hence, (F2a) holds.

Lemma 17. Let $\langle F, A\rangle \in \mathscr{F} \mathcal{S}(H, E)$. Then, one has the following.

(1) (F3a) holds if and only if the following condition holds: (F3b) for all $y, y_{1}^{n-1} \in H$ and $\varepsilon \in A$, there exists $x \in H$ such that

$$
\begin{aligned}
\min & \left\{F(\varepsilon)_{y_{1}}^{y_{n-1}}, F(\varepsilon)(y)\right\}_{y} \\
& \subseteq \vee q_{(\gamma, \delta)} \mathscr{F}\left(F(\varepsilon)(x)_{x}, F(\varepsilon)\left(y_{1}\right)_{y_{1}}, \ldots, F(\varepsilon)\left(y_{n-1}\right)_{y_{n-1}}\right) .
\end{aligned}
$$

(2) If (F3a) holds, then the following condition holds:

(F3c) for all $y_{1}^{n-1} \in H$ and $\varepsilon \in A$,

$$
\begin{aligned}
F(\varepsilon) & \cap \min \left\{F(\varepsilon)_{y_{1}}^{y_{n-1}}\right\}_{H} \\
\subseteq \vee q_{(\gamma, \delta)} & \mathscr{F}\left(F(\varepsilon), F(\varepsilon)\left(y_{1}\right)_{y_{1}}, \ldots, F(\varepsilon)\left(y_{n-1}\right)_{y_{n-1}}\right) .
\end{aligned}
$$

Moreover, (F3c) implies (F3a) if $\langle F, A\rangle$ has the sup-property or $H$ is finite.

Proof. (F3a) $\Rightarrow$ (F3b) Let $y, y_{1}^{n-1} \in H$ and $\varepsilon \in A$. By (F3a), there exists $x \in H$ such that

$$
\begin{gathered}
y \in f\left(x, y_{1}^{n-1}\right), \\
\max \{F(\varepsilon)(x), \gamma\} \geq \min \left\{F(\varepsilon)_{y_{1}}^{y_{n-1}}, F(\varepsilon)(y), \delta\right\} .
\end{gathered}
$$

This implies $\min \left\{\max \left\{F(\varepsilon)_{y_{1}}^{y_{n-1}}, \gamma\right\}, \max \{F(\varepsilon)(x), \gamma\}\right\} \quad \geq$ $\min \left\{F(\varepsilon)_{y_{1}}^{y_{n-1}}, F(\varepsilon)(y), \delta\right\}$, and so

$$
\begin{aligned}
\max & \left\{\mathscr{F}\left(F(\varepsilon)(x)_{x}, F(\varepsilon)\left(y_{1}\right)_{y_{1}}, \ldots, F(\varepsilon)\left(y_{n-1}\right)_{y_{n-1}}\right)(y), \gamma\right\} \\
& =\max \left\{\left(\min \left\{F(\varepsilon)_{y_{1}}^{y_{n-1}}, F(\varepsilon)(x)\right\}_{f\left(x, y_{1}^{n-1}\right)}\right)(y), \gamma\right\} \\
& \geq \min \left\{F(\varepsilon)_{y_{1}}^{y_{n-1}}, F(\varepsilon)(y), \delta\right\} \\
& =\min \left\{\left(\min \left\{F(\varepsilon)_{y_{1}}^{y_{n-1}}, F(\varepsilon)(y)\right\}\right)_{y}(y), \delta\right\} .
\end{aligned}
$$

It follows that $\min \left\{F(\varepsilon)_{y_{1}}^{y_{n-1}}, F(\varepsilon)(y)\right\}_{y} \subseteq \vee q_{(\gamma, \delta)} \mathscr{F}\left(F(\varepsilon)(x)_{x}\right.$, $\left.F(\varepsilon)\left(y_{1}\right)_{y_{1}}, \ldots, F(\varepsilon)\left(y_{n-1}\right)_{y_{n-1}}\right)$ and so (F3b) holds.

$(\mathrm{F} 3 \mathrm{~b}) \Rightarrow(\mathrm{F} 3 \mathrm{a})$ Let $y, y_{1}^{n-1} \in H$. By (F3b), there exists $x \in$ $H$ such that

$$
\begin{aligned}
& \min \left\{F(\varepsilon)_{y_{1}}^{y_{n-1}}, F(\varepsilon)(y)\right\}_{y} \\
& \quad \subseteq \vee q_{(\gamma, \delta)} \mathscr{F}\left(F(\varepsilon)(x)_{x}, F(\varepsilon)\left(y_{1}\right)_{y_{1}}, \ldots, F(\varepsilon)\left(y_{n-1}\right)_{y_{n-1}}\right) .
\end{aligned}
$$

It follows from Lemma 2 that

$$
\begin{aligned}
& \max \left\{\left(\min \left\{F(\varepsilon)_{y_{1}}^{y_{n-1}}, F(\varepsilon)(x)\right\}_{f\left(x, y_{1}^{n-1}\right)}\right)(y), \gamma\right\} \\
&=\max \left\{\mathscr{F}\left(F(\varepsilon)(x)_{x}, F(\varepsilon)\left(y_{1}\right)_{y_{1}}, \ldots, F(\varepsilon)\left(y_{n-1}\right)_{y_{n-1}}\right)\right. \\
&\times(y), \gamma\} \\
& \geq \min \left\{\left(\min \left\{F(\varepsilon)_{y_{1}}^{y_{n-1}}, F(\varepsilon)(y)\right\}\right)_{y}(y), \delta\right\} \\
&=\min \left\{F(\varepsilon)_{y_{1}}^{y_{n-1}}, F(\varepsilon)(y), \delta\right\} .
\end{aligned}
$$

This implies $y \in f\left(x, y_{1}^{n-1}\right)$ and $\max \left\{\min \left\{F(\varepsilon)_{y_{1}}^{y_{n-1}}, F(\varepsilon)(x)\right\}\right.$, $\gamma\} \geq \min \left\{F(\varepsilon)_{y_{1}}^{y_{n-1}}, F(\varepsilon)(y), \delta\right\}$. Hence,

$$
\begin{aligned}
\max \{F(\varepsilon)(x), \gamma\} & \geq \max \left\{\min \left\{F(\varepsilon)_{y_{1}}^{y_{n-1}}, F(\varepsilon)(x)\right\}, \gamma\right\} \\
& \geq \min \left\{F(\varepsilon)_{y_{1}}^{y_{n-1}}, F(\varepsilon)(y), \delta\right\}
\end{aligned}
$$

and so (F3a) holds.

$(\mathrm{F} 3 \mathrm{a}) \Rightarrow(\mathrm{F} 3 \mathrm{c})$ This is straightforward by $(\mathrm{F} 3 \mathrm{~b})$. 
In the following, assume that $\langle F, A\rangle$ has the sup-property or $H$ is finite. We prove (F3c) $\Rightarrow(\mathrm{F} 3 \mathrm{a})$. Let $y_{1}^{n-1} \in H$. If, for any $x \in H$ such that $y \in f\left(x, y_{1}^{n-1}\right)$, we have

$$
\begin{aligned}
F(\varepsilon)(x) & \leq \max \{F(\varepsilon)(x), \gamma\} \\
& <\min \left\{F(\varepsilon)_{y_{1}}^{y_{n-1}}, F(\varepsilon)(y), \delta\right\} \leq F(\varepsilon)_{y_{1}}^{y_{n-1}},
\end{aligned}
$$

then since $\langle F, A\rangle$ has the sup-property or $H$ is finite, we have

$$
\begin{aligned}
\max & \left\{\mathscr{F}\left(F(\varepsilon), F(\varepsilon)\left(y_{1}\right)_{y_{1}}, \ldots, F(\varepsilon)\left(y_{n-1}\right)_{y_{n-1}}\right)(y), \gamma\right\} \\
= & \max \left\{\sup _{y \in f\left(x, y_{1}^{n-1}\right)} \min \left\{F(\varepsilon)(x), F(\varepsilon)_{y_{1}}^{y_{n-1}}\right\}, \gamma\right\} \\
= & \max \left\{\sup _{y \in f\left(x, y_{1}^{n-1}\right)} F(\varepsilon)(x), \gamma\right\} \\
= & \sup _{y \in f\left(x, y_{1}^{n-1}\right)} \max \{F(\varepsilon)(x), \gamma\} \\
< & \min \left\{F(\varepsilon)_{y_{1}}^{y_{n-1}}, F(\varepsilon)(y), \delta\right\} \\
= & \min \left\{\left(F(\varepsilon) \cap \min \left\{F(\varepsilon)_{y_{1}}^{y_{n-1}}\right\}_{H}\right)(y), \delta\right\},
\end{aligned}
$$

which contradicts $F(\varepsilon) \cap \min \left\{F(\varepsilon)_{y_{1}}^{y_{n-1}}\right\}_{H} \subseteq \vee q_{(\gamma, \delta)} \mathscr{F}(F(\varepsilon)$, $\left.F(\varepsilon)\left(y_{1}\right)_{y_{1}}, \ldots, F(\varepsilon)\left(y_{n-1}\right)_{y_{n-1}}\right)$ by Lemma 2. Hence (F3a) holds.

Lemma 18. Let $\langle F, A\rangle \in \mathscr{F} \mathcal{S}(H, E)$. Then (F4a) holds if and only if the following condition holds:

(F4c) for all $x, y \in H, \varepsilon \in A$, and $r \in(\gamma, 1], r_{x} \in \mathscr{F}\left(r_{y}\right.$, $\underbrace{F(\varepsilon), \ldots, F(\varepsilon)}_{n-1})$ implies $r_{y} \epsilon_{\gamma} \vee q_{\delta} \mathscr{F}(r_{x}, \underbrace{F(\varepsilon), \ldots, F(\varepsilon)}_{n-1})$.

Proof. (F4a) $\Rightarrow$ (F4c) Let $x, y \in H$ and $r \in(\gamma, 1]$. If $r_{y} \epsilon_{\gamma} \mathscr{F}(r_{x}, \underbrace{F(\varepsilon), \ldots, F(\varepsilon)}_{n-1})$ but $r_{x} \overline{\epsilon_{\gamma} \vee q_{\delta}} \mathscr{F}(r_{y}, \underbrace{F(\varepsilon), \ldots, F(\varepsilon)}_{n-1})$.

Then

$$
\begin{aligned}
& \mathscr{F}(x, \underbrace{F(\varepsilon), \ldots, F(\varepsilon)}_{n-1})(y) \\
& \quad \geq \mathscr{F}(r_{x}, \underbrace{F(\varepsilon), \ldots, F(\varepsilon)}_{n-1}) \geq r>\gamma,
\end{aligned}
$$

but $\mathscr{F}(r_{y}, \underbrace{F(\varepsilon), \ldots, F(\varepsilon)}_{n-1})(x) \quad<\quad r \quad$ and $\mathscr{F}(r_{y}, \underbrace{F(\varepsilon), \ldots, F(\varepsilon)}_{n-1})(x)+r \leq 2 \delta$. Then

$$
\begin{gathered}
\min \{\mathscr{F}(y, \underbrace{F(\varepsilon), \ldots, F(\varepsilon)}_{n-1})(x), r\} \\
=\mathscr{F}(r_{y}, \underbrace{F(\varepsilon), \ldots, F(\varepsilon)}_{n-1})(x)<r
\end{gathered}
$$

gives $F(y, \underbrace{\mu, \ldots, \mu}_{n-1})(x)<r$ and so $2 \mathscr{F}(y, \underbrace{F(\varepsilon), \ldots, F(\varepsilon)}_{n-1})(x)$ $<\min \{\mathscr{F}(y, \underbrace{F(\varepsilon), \ldots, F(\varepsilon)})(x), r\}+r=\mathscr{F}\left(r_{y}\right.$, $\underbrace{F(\varepsilon), \ldots, F(\varepsilon)}_{n-1})(x)+r \leq 2 \delta$; that is, $\mathscr{F}(y, \underbrace{F(\varepsilon), \ldots, F(\varepsilon)}_{n-1})(x)<$ $\delta$. Thus we have $\max \{\mathscr{F}(y, \underbrace{F(\varepsilon), \ldots, F(\varepsilon)})(x), \gamma\}<\min \{\mathscr{F}(x$, $\underbrace{F(\varepsilon), \ldots, F(\varepsilon)}_{n-1})(y), \delta\}$, a contradiction. Hence (F4c) holds.

$(\mathrm{F} 4 \mathrm{c}) \Rightarrow(\mathrm{F} 4 \mathrm{a})$ Let $x, y \in H$. If there exists $r$ such that $\max \{\mathscr{F}(y, \underbrace{F(\varepsilon), \ldots, F(\varepsilon)}_{n-1})(x), \gamma\}<r<$ $\min \{\mathscr{F}(x, \underbrace{F(\varepsilon), \ldots, F(\varepsilon)}_{n-1})(y), \delta\}$, then

$$
\begin{aligned}
\mathscr{F} & (r_{y}, \underbrace{F(\varepsilon), \ldots, F(\varepsilon)}_{n-1})(x) \\
& =\min \{\mathscr{F}(y, \underbrace{F(\varepsilon), \ldots, F(\varepsilon)}_{n-1})(x), r\}<r \\
& \leq \min \{\mathscr{F}(x, \underbrace{F(\varepsilon), \ldots, F(\varepsilon)}_{n-1})(y), r\} \\
& =\mathscr{F}(r_{x}, \underbrace{F(\varepsilon), \ldots, F(\varepsilon)}_{n-1})(y)
\end{aligned}
$$

and $\gamma<r<\delta$. This implies that $r_{y} \in \mathscr{F}(r_{x}, \underbrace{F(\varepsilon), \ldots, F(\varepsilon)}_{n-1})$ but $\quad r_{x} \overline{\epsilon_{\gamma} \vee q_{\delta}} \mathscr{F}(r_{y}, \underbrace{F(\varepsilon), \ldots, F(\varepsilon)}_{n-1}), \quad$ a contradiction. Hence $\max \{\mathscr{F}(y, \underbrace{F(\varepsilon), \ldots, F(\varepsilon)}_{n-1})(x), \gamma\} \geq \min \{\mathscr{F}(x$, $\underbrace{F(\varepsilon), \ldots, F(\varepsilon)})(y), \delta\} \quad$ and so $\max \{\min \{\mathscr{F}(y$, $\underbrace{F(\varepsilon), \ldots, F(\varepsilon)})(x), \delta\}, \gamma\} \geq \max \{\min \{\mathscr{F}(x, \underbrace{F(\varepsilon), \ldots, F(\varepsilon)})(y)$, $\delta\}, \gamma\} .^{n-1}$ In a similar way, $\quad$ we have $\max \{\min \{\mathscr{F}(x, \underbrace{F(\varepsilon), \ldots, F(\varepsilon)})(y), \delta\}, \gamma\} \geq \max \{\min \{\mathscr{F}(y$, $\underbrace{F(\varepsilon), \ldots, F(\varepsilon)})(x), \delta\}, \gamma\}$. Thus (F4a) holds.

For any fuzzy soft set $\langle F, A\rangle$ over $H, \varepsilon \in A$, and $r \in(\gamma, 1]$, denote $F(\varepsilon)_{r}=\left\{x \in H \mid x_{r} \epsilon_{\gamma} F(\varepsilon)\right\},\langle F(\varepsilon)\rangle_{r}=\{x \in H \mid$ $\left.x_{r} q_{\delta} F(\varepsilon)\right\}$, and $[F(\varepsilon)]_{r}=\left\{x \in H \mid x_{r} \epsilon_{\gamma} \vee q_{\delta} F(\varepsilon)\right\}$. The next theorem presents the relationships between $\left(\epsilon_{\gamma}, \epsilon_{\gamma} \vee q_{\delta}\right)$ fuzzy soft (invertible) $n$-ary subhypergroups over $H$ and crisp (invertible) $n$-ary subhypergroups of $H$.

Theorem 19. Let $\langle F, A\rangle \in \mathscr{F} \mathcal{S}(H, E)$. Then,

(1) $\langle F, A\rangle$ is an $\left(\epsilon_{\gamma}, \epsilon_{\gamma} \vee q_{\delta}\right)$-fuzzy soft (invertible) $n$-ary subhypergroup over $H$ if and only if nonempty subset $F(\varepsilon)_{r}$ is an (invertible) n-ary subhypergroup of $H$ for all $\varepsilon \in A$ and $r \in(\gamma, \delta]$;

(2) if $2 \delta=1+\gamma$, then $\langle F, A\rangle$ is an $\left(\epsilon_{\gamma}, \epsilon_{\gamma} \vee q_{\delta}\right)$-fuzzy soft (invertible) n-ary subhypergroup over $H$ if and only if nonempty subset $\langle F(\varepsilon)\rangle_{r}$ is an (invertible) n-ary subhypergroup of $H$ for all $\varepsilon \in A$ and $r \in(\delta, 1]$; 
(3) $\langle F, A\rangle$ is an $\left(\epsilon_{\gamma}, \epsilon_{\gamma} \vee q_{\delta}\right)$-fuzzy soft (invertible) n-ary subhypergroup over $H$ if and only if nonempty subset $[F(\varepsilon)]_{r}$ is an (invertible) $n$-ary subhypergroup of $H$ for all $\varepsilon \in A$ and $r \in(\gamma, \min \{2 \delta-\gamma, 1\}]$.

Proof. We only prove (2) and (3). (1) can be easily proved.

(2) Assume that $2 \delta=1+\gamma$. Let $\langle F, A\rangle$ be an $\left(\epsilon_{\gamma}, \epsilon_{\gamma} \vee\right.$ $\left.q_{\delta}\right)$-fuzzy soft $n$-ary subhypergroup over $H$ and assume that $\langle F(\varepsilon)\rangle_{r} \neq \emptyset$ for some $\varepsilon \in A$ and $r \in(\delta, 1]$. Let $x_{1}^{n} \in$ $\langle F(\varepsilon)\rangle_{r}$. Then $r_{x_{1}} q_{\delta} F(\varepsilon), \ldots, r_{x_{n}} q_{\delta} F(\varepsilon)$; that is, $F(\varepsilon)\left(x_{1}\right)+r>$ $2 \delta, \ldots, F(\varepsilon)\left(x_{n}\right)+r>2 \delta$. Since $\langle F, A\rangle$ is an $\left(\epsilon_{\gamma}, \epsilon_{\gamma} \vee q_{\delta}\right)$-fuzzy $n$-ary subhypergroup over $H$, we have $\max \{F(\varepsilon)(x), \gamma\} \geq$ $\min \left\{F(\varepsilon)_{x_{1}}^{x_{n}}, \delta\right\}$ for all $x \in f\left(x_{1}^{n}\right)$. Hence, by $r>\delta$,

$$
\begin{aligned}
\max & \{F(\varepsilon)(x)+r, \gamma+r\} \\
= & \max \{F(\varepsilon)(x), \gamma\}+r \geq \min \left\{F(\varepsilon)_{x_{1}}^{x_{n}}, \delta\right\}+r \\
= & \min \left\{F(\varepsilon)\left(x_{1}\right)+r, \ldots, F(\varepsilon)\left(x_{n}\right)+r, \delta+r\right\}>2 \delta .
\end{aligned}
$$

From $r \leq 1=2 \delta-\gamma$, that is, $r+\gamma \leq 2 \delta$, we have $F(\varepsilon)(x)+r>2 \delta$ and so $x \in\langle F(\varepsilon)\rangle_{r}$. Similarly we can show that $e \in\langle F(\varepsilon)\rangle_{r}$ and, for all $y, y_{1}^{n-1} \in\langle F(\varepsilon)\rangle_{r}$, there exists $x \in\langle F(\varepsilon)\rangle_{r}$ such that $y \in f\left(x, y_{1}^{n-1}\right)$. Therefore, $\langle F(\varepsilon)\rangle_{r}$ is an $n$-ary hypergroup of $H$.

Conversely, assume that the given condition holds. Let $x_{1}^{n} \in H$ and $\varepsilon \in A$. If there exists $x \in f\left(x_{1}^{n}\right)$ such that $\max \{F(\varepsilon)(x), \gamma\}<\min \left\{F(\varepsilon)_{x_{1}}^{x_{n}}, \delta\right\}$, take $r=2 \delta-$ $\max \{F(\varepsilon)(x), \gamma\}$. Then $r \in(\delta, 1], F(\varepsilon)(x) \leq 2 \delta-r, F(\varepsilon)\left(x_{1}\right)>$ $\max \{G(\varepsilon)(x), \gamma\}=2 \delta-r, \ldots, F(\varepsilon)\left(x_{n}\right)>\max \{G(\varepsilon)(x), \gamma\}=$ $2 \delta-r$, that is, $x_{1}^{n} \in\langle F(\varepsilon)\rangle_{r}$, but $x \notin\langle F(\varepsilon)\rangle_{r}$, a contradiction. Hence $\max \{F(\varepsilon)(x), \gamma\} \geq \min \left\{F(\varepsilon)_{x_{1}}^{x_{n}}, \delta\right\}$ and so $\max \left\{\inf _{x \in f\left(x_{1}^{n}\right)} F(\varepsilon)(x), \gamma\right\} \geq \min \left\{F(\varepsilon)_{x_{1}}^{x_{n}}, \delta\right\}$. Similarly we can show that conditions (F1a) and (F3a) hold. Therefore, $\langle F, A\rangle$ is an $\left(\epsilon_{\gamma}, \epsilon_{\gamma} \vee q_{\delta}\right)$-fuzzy soft $n$-ary subhypergroup over $H$.

(3) Let $\langle F, A\rangle$ be an $\left(\epsilon_{\gamma}, \epsilon_{\gamma} \vee q_{\delta}\right)$-fuzzy soft $n$-ary subhypergroup over $H$ and assume that $[F(\varepsilon)]_{r} \neq \emptyset$ for some $\varepsilon \in A$ and $r \in(\gamma, \min \{2 \delta-\gamma, 1\}]$. Let $x_{1}^{n} \in[F(\varepsilon)]_{r}$. Then $r_{x_{1}} \epsilon_{\gamma} \vee q_{\delta} F(\varepsilon), \ldots, r_{x_{n}} \epsilon_{\gamma} \vee q_{\delta} F(\varepsilon)$; that is, $F(\varepsilon)\left(x_{1}\right) \geq r>\gamma$ or $F(\varepsilon)\left(x_{1}\right)>2 \delta-r \geq 2 \delta-(2 \delta-\gamma)=\gamma, \ldots, F(\varepsilon)\left(x_{n}\right) \geq r>\gamma$ or $F(\varepsilon)\left(x_{n}\right)>2 \delta-r \geq 2 \delta-(2 \delta-\gamma)=\gamma$. Since $\langle F, A\rangle$ is an $\left(\epsilon_{\gamma}, \epsilon_{\gamma} \vee q_{\delta}\right)$-fuzzy $n$-ary subhypergroup over $H$, we have $\max \{F(\varepsilon)(x), \gamma\} \geq \min \left\{F(\varepsilon)_{x_{1}}^{x_{n}}, \delta\right\}$ for all $x \in f\left(x_{1}^{n}\right)$ and so $F(\varepsilon)(x) \geq \min \left\{F(\varepsilon)_{x_{1}}^{x_{n}}, \delta\right\}$ since $\gamma<\min \left\{F(\varepsilon)_{x_{1}}^{x_{n}}, \delta\right\}$ in any case. Now we consider the following cases.

Case $1(r \in(\gamma, \delta])$. In this case, $2 \delta-r \geq \delta \geq r$.

(1) If $F(\varepsilon)\left(x_{1}\right) \geq r$ or $\cdots$ or $F(\varepsilon)\left(x_{n}\right) \geq r$, then $F(\varepsilon)(x) \geq$ $\min \left\{F(\varepsilon)_{x_{1}}^{x_{n}}, \delta\right\} \geq r$. Hence $z_{r} \in_{\gamma} F(\varepsilon)$.

(2) If $F(\varepsilon)\left(x_{i}\right)+r>2 \delta$, for all $1 \leq i \leq n$, then $F(\varepsilon)(x) \geq$ $\min \left\{F(\varepsilon)_{x_{1}}^{x_{n}}, \delta\right\}=\delta \geq r$. Hence $z_{r} \in_{\gamma} F(\varepsilon)$.

Case $2(r \in(\delta, \min \{2 \delta-\gamma, 1\}])$. In this case, $r>\delta>2 \delta-r$.

(1) If $F(\varepsilon)\left(x_{i}\right) \geq r$, for all $1 \leq i \leq n$, then $F(\varepsilon)(x) \geq$ $\min \left\{F(\varepsilon)_{x_{1}}^{x_{n}}, \delta\right\}=\delta>2 \delta-r$. Hence $z_{r} q_{\delta} F(\varepsilon)$.
(2) If $F(\varepsilon)\left(x_{1}\right)+r>2 \delta$ or $\cdots$ or $F(\varepsilon)\left(x_{n}\right)+r>2 \delta$, then $F(\varepsilon)(x) \geq \min \left\{F(\varepsilon)_{x_{1}}^{x_{n}}, \delta\right\}>2 \delta-r$. Hence $z_{r} q_{\delta} F(\varepsilon)$.

Thus, in any case, $x_{r} \in_{\gamma} \vee q_{\delta} F(\varepsilon)$; that is, $x \in[F(\varepsilon)]_{r}$. Similarly, we can show that $e \in[F(\varepsilon)]_{r}$ and, for all $y, y_{1}^{n-1} \in$ $[F(\varepsilon)]_{r}$, there exists $x \in[F(\varepsilon)]_{r}$ such that $y \in f\left(x, y_{1}^{n-1}\right)$. Therefore, $[F(\varepsilon)]_{r}$ is an $n$-ary hypergroup of $H$.

Conversely, assume that the given condition holds. Let $x_{1}^{n} \in H$ and $\varepsilon \in A$. If there exists $x \in f\left(x_{1}^{n}\right)$ such that $\max \{F(\varepsilon)(x), \gamma\}<r=\min \left\{F(\varepsilon)_{x_{1}}^{x_{n}}, \delta\right\}$, then $F(\varepsilon)\left(x_{1}\right) \geq$ $r>\gamma, \ldots, F(\varepsilon)\left(x_{n}\right) \geq r>\gamma, F(\varepsilon)(x)<r$, and $F(\varepsilon)(x)+$ $r<2 r \leq 2 \delta$, that is, $\left(x_{1}\right)_{r} \in_{\gamma} F(\varepsilon), \ldots,\left(x_{n}\right)_{r} \in_{\gamma} F(\varepsilon)$, but $x_{r} \overline{\epsilon_{\gamma} \vee q_{\delta}} F(\varepsilon)$, that is, $x_{1}^{n} \in[F(\varepsilon)]_{r}$, but $x \notin[F(\varepsilon)]_{r}$, a contradiction. Hence $\max \{F(\varepsilon)(x), \gamma\} \geq \min \left\{F(\varepsilon)_{x_{1}}^{x_{n}}, \delta\right\}$ and so $\max \left\{\inf _{x \in f\left(x_{1}^{n}\right)} F(\varepsilon)(x), \gamma\right\} \geq \min \left\{F(\varepsilon)_{x_{1}}^{x_{n}}, \delta\right\}$. Similarly, we can show that conditions (F1a) and (F3a) hold. Therefore, $\langle F, A\rangle$ is an $\left(\epsilon_{\gamma}, \epsilon_{\gamma} \vee q_{\delta}\right)$-fuzzy soft $n$-ary subhypergroup over $H$.

As a direct consequence of Theorem 19, we have the following results.

Corollary 20. Let $\gamma, \gamma^{\prime}, \delta, \delta^{\prime} \in[0,1]$ be such that $\gamma<\delta$, $\gamma^{\prime}<\delta^{\prime}, \gamma<\gamma^{\prime}$, and $\delta^{\prime}<\delta$. Then every $\left(\epsilon_{\gamma}, \epsilon_{\gamma} \vee q_{\delta}\right)$-fuzzy (invertible) n-ary soft subhypergroup over $H$ is an $\left(\epsilon_{\gamma^{\prime}}, \epsilon_{\gamma^{\prime}} \vee\right.$ $q_{\delta^{\prime}}$ )-fuzzy soft (invertible) n-ary subhypergroup over $H$.

Corollary 21. Let $P \in \mathscr{P}^{*}(H)$. Then $P$ is an (invertible) n-ary subhypergroup of $H$ if and only if $\Sigma(P, A, r)$ is an $\left(\epsilon_{\gamma}, \epsilon_{\gamma} \vee q_{\delta}\right)$ fuzzy soft (invertible) $n$-ary subhypergroup over $H$ for any $A \subseteq$ $E$ and $r \geq \delta$.

Lemma 22. Let $\langle F, A\rangle \in \mathscr{F} \mathcal{S}(H, E)$. Then

$$
\begin{gathered}
\langle F, A\rangle \epsilon_{(\gamma, \delta)} \mathfrak{F}(\underbrace{\left\langle\widetilde{1_{e}}, A\right\rangle, \ldots,\left\langle\widetilde{1_{e}}, A\right\rangle,\langle F, A\rangle,}_{i-1} \\
\underbrace{\left\langle\widetilde{1_{e}}, A\right\rangle, \ldots,\left\langle\widetilde{1_{e}}, A\right\rangle}_{n-i})
\end{gathered}
$$

for all $1 \leq i \leq n$

Proof. It is straightforward.

Lemma 23. Let $\langle F, A\rangle$ be an $\left(\epsilon_{\gamma}, \epsilon_{\gamma} \vee q_{\delta}\right)$-fuzzy soft n-ary subhypergroup over $H$. Then

(1) $\mathfrak{F}(\underbrace{\langle F, A\rangle, \ldots,\langle F, A\rangle}_{i}, \underbrace{\left\langle\widetilde{1_{e}}, A\right\rangle, \ldots,\left\langle\widetilde{1_{e}}, A\right\rangle}_{n-i}) \asymp_{(\gamma, \delta)}\langle F, A\rangle$ for all $1 \leq i \leq n$;

(2) $\mathfrak{F}(\left\langle\widetilde{1_{x}}, A\right\rangle, \underbrace{\langle F, A\rangle, \ldots,\langle F, A\rangle}_{i}$,

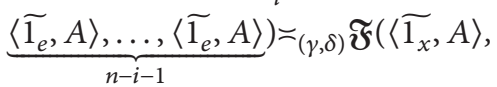

$\langle F, A\rangle, \ldots,\langle F, A\rangle)$ for all $x \in H$ and $1 \leq i \leq n$. 
Proof. (1) Let $\langle F, A\rangle$ be an $\left(\epsilon_{\gamma}, \epsilon_{\gamma} \vee q_{\delta}\right)$-fuzzy soft $n$-ary subhypergroup over $H, \varepsilon \in A$, and $1 \leq i \leq n$. Since $\max \{F(\varepsilon)(e), \gamma\} \geq \min \{F(\varepsilon)(x), \delta\}$, for all $x \in H$, we have

$$
\begin{aligned}
F(\varepsilon) & \subseteq \vee q_{(\gamma, \delta)} \mathscr{F}(F(\varepsilon), \underbrace{1_{e}, \ldots, 1_{e}}_{n-1}) \\
& \subseteq \vee q_{(\gamma, \delta)} \mathscr{F}(\underbrace{F(\varepsilon), \ldots, F(\varepsilon)}_{i}, \underbrace{1_{e}, \ldots, 1_{e}}_{n-i}) \\
& \subseteq \vee q_{(\gamma, \delta)} \mathscr{F}(\underbrace{F(\varepsilon), \ldots, F(\varepsilon)}_{n}) \subseteq \vee q_{(\gamma, \delta)} F(\varepsilon) .
\end{aligned}
$$

Therefore, $\quad \mathscr{F}(F(\varepsilon), \underbrace{1_{e}, \ldots, 1_{e}}_{n-1})={ }_{(\gamma, \delta)} F(\varepsilon) \quad$ and $\quad$ so

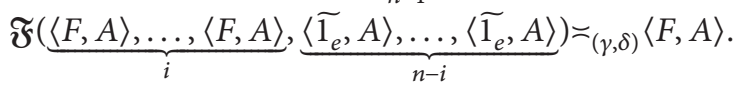

(2) Let $x \in H$ and $1 \leq i \leq n$. By (1), we have

$$
\begin{aligned}
& \mathfrak{\mho}(\left\langle\widetilde{1_{x}}, A\right\rangle, \underbrace{\langle F, A\rangle, \ldots,\langle F, A\rangle}_{n-1}) \\
& \asymp_{(\gamma, \delta)} \mathfrak{\mho}(\left\langle\widetilde{1_{x}}, A\right\rangle, \underbrace{\langle F, A\rangle, \ldots,\langle F, A\rangle}_{n-2}, \\
& \left.\mathfrak{F}(\langle F, A\rangle, \underbrace{\left\langle\widetilde{1_{e}}, A\right\rangle, \ldots,\left\langle\widetilde{1_{e}}, A\right\rangle}_{n-1})\right) \\
& =_{(\gamma, \delta)} \mathfrak{F}(\left\langle\widetilde{1_{x}}, A\right\rangle, \underbrace{\langle F, A\rangle, \ldots,\langle F, A\rangle}_{i-1}, \\
& \mathfrak{F}(\underbrace{\langle F, A\rangle, \ldots,\langle F, A\rangle}_{n-i}, \underbrace{\left\langle\widetilde{1_{e}}, A\right\rangle, \ldots,\left\langle\widetilde{1_{e}}, A\right\rangle}_{i}), \\
& \underbrace{\left\langle\widetilde{1_{e}}, A\right\rangle, \ldots,\left\langle\widetilde{1_{e}}, A\right\rangle}_{n-i-1}) \\
& =_{(\gamma, \delta)} \mathfrak{F}(\left\langle\widetilde{1}_{x}, A\right\rangle, \underbrace{\langle F, A\rangle, \ldots,\langle F, A\rangle}_{i-1}, \\
& \langle F, A\rangle, \underbrace{\left\langle\widetilde{1_{e}}, A\right\rangle, \ldots,\left\langle\widetilde{1_{e}}, A\right\rangle}_{n-i-1}) \\
& \asymp_{(\gamma, \delta)} \mathfrak{F}(\left\langle\widetilde{1}_{x}, A\right\rangle, \underbrace{\langle F, A\rangle, \ldots,\langle F, A\rangle}_{i},
\end{aligned}
$$

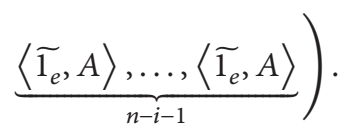

This completes the proof.
Lemma 24. Let $\langle F, A\rangle$ be an $\left(\epsilon_{\gamma}, \epsilon_{\gamma} \vee q_{\delta}\right)$-fuzzy soft n-ary subhypergroup over $H$. Then $\langle F, A\rangle$ is invertible if and only if

$$
\begin{aligned}
& \max \left\{\min \left\{\mathscr{F}(x, \underbrace{F(\varepsilon), \ldots, F(\varepsilon)}_{i}, e^{*})(y), \delta\right\}, \gamma\right\} \\
& =\max \left\{\min \left\{\mathscr{F}(y, \underbrace{F(\varepsilon), \ldots, F(\varepsilon)}_{i}, e^{*})(x), \delta\right\}, \gamma\right\}
\end{aligned}
$$

for all $x, y \in H, \varepsilon \in A$, and $1 \leq i \leq n$. Proof It is straightforward by Lemmas 18 and 23.

Lemma 25. Let $\langle F, A\rangle,\left\langle F_{1}, A\right\rangle, \ldots,\left\langle F_{i}, A\right\rangle(2 \leq i \leq n)$ be $\left(\epsilon_{\gamma}, \epsilon_{\gamma} \vee q_{\delta}\right)$-fuzzy soft invertible n-ary subhypergroups over $H$. Then

(1) $\max \left\{\min \left\{\mathscr{F}\left(x, F_{1}^{i}(\varepsilon), e *\right)(y), \delta\right\}, \gamma\right\}=$ $\max \left\{\min \left\{\mathscr{F}\left(y, F_{1}^{i}(\varepsilon), e *\right)(x), \delta\right\}, \gamma\right\}$, for all $x, y \in H$ and $\varepsilon \in A$;

(2) for all $x, y, y_{1}^{n-1} \in H$ such that $y \in f\left(x, y_{1}^{n-1}\right)$ and $\varepsilon \in A$, one has $\max \{F(\varepsilon)(x), \gamma\} \geq$ $\min \left\{F(\varepsilon)_{y_{1}}^{y_{n-1}}, F(\varepsilon)(y), \delta\right\}$.

Proof. (1) Let $x, y \in H$ and $\varepsilon \in A$. Then, by Lemma 23, we have

$$
\begin{aligned}
\mathscr{F} & \left(x, F_{1}^{i}(\varepsilon), e *\right)=\mathscr{F}\left(x, F_{1}(\varepsilon), F_{2}(\varepsilon), F_{3}^{i}(\varepsilon), e *\right) \\
{ }_{(\gamma, \delta)} & \mathscr{F}\left(x, F_{1}(\varepsilon), \mathscr{F}(\underbrace{e, \ldots, e}_{n-2}, F_{2}(\varepsilon), e), F_{3}^{i}(\varepsilon), e *\right) \\
{ }_{(\gamma, \delta)} & \mathscr{F}\left(\mathscr{F}\left(x, F_{1}(\varepsilon), e *\right), F_{2}(\varepsilon), e, F_{3}^{i}(\varepsilon), e *\right) \\
{ }_{(\gamma, \delta)} & \mathscr{F}\left(\mathscr{F}\left(x, F_{1}(\varepsilon), e *\right), F_{2}^{i}(\varepsilon), e *\right) \\
{ }_{(\gamma, \delta)} & \mathscr{F}\left(\mathscr{F}\left(x, F_{1}(\varepsilon), e *\right), \mathscr{F}\left(F_{2}^{i}(\varepsilon), e *\right), e *\right) .
\end{aligned}
$$

It follows that

$$
\begin{gathered}
\max \left\{\min \left\{\mathscr{F}\left(x, F_{1}^{i}(\varepsilon), e *\right)(y), \delta\right\}, \gamma\right\} \\
\geq \min \left\{\operatorname { m a x } \left\{\mathscr { F } \left(\mathscr{F}\left(x, F_{1}(\varepsilon), e *\right),\right.\right.\right. \\
\left.\left.\left.\mathscr{F}\left(F_{2}^{i}(\varepsilon), e *\right), e *\right)(y), \gamma\right\}, \delta\right\} \\
=\min \left\{\operatorname { m a x } \left\{\operatorname { s u p } _ { y \in f ( a _ { 1 } , a _ { 2 } , e * ) } \operatorname { m i n } \left\{\mathscr{F}\left(x, F_{1}(\varepsilon), e *\right)\left(a_{1}\right),\right.\right.\right. \\
\gamma\}, \delta\} \\
\left.\mathscr{F}\left(F_{2}^{i}(\varepsilon), e *\right)\left(a_{2}\right)\right\},
\end{gathered}
$$




$$
\begin{aligned}
& =\sup _{z_{1} \in H} \min \left\{\operatorname { m a x } \left\{\operatorname { m i n } \left\{\mathscr{F}\left(x, F_{1}(\varepsilon), e *\right)\left(z_{1}\right),\right.\right.\right. \\
& \left.\left.\left.\mathscr{F}\left(z_{1}, F_{2}^{i}(\varepsilon), e *\right)(y)\right\}, \gamma\right\}, \delta\right\} \\
& =\sup _{z_{1}, z_{2} \in H} \min \left\{\operatorname { m a x } \left\{\operatorname { m i n } \left\{\mathscr{F}\left(x, F_{1}(\varepsilon), e *\right)\left(z_{1}\right),\right.\right.\right. \\
& \mathscr{F}\left(z_{1}, F_{2}(\varepsilon), e *\right)\left(z_{2}\right), \\
& \left.\left.\left.\mathscr{F}\left(z_{2}, F_{3}^{i}(\varepsilon), e *\right)(y)\right\}, \gamma\right\}, \delta\right\} \\
& =\cdots \\
& =\sup _{z_{1}^{i} \in H} \min \left\{\operatorname { m a x } \left\{\operatorname { m i n } \left\{\mathscr{F}\left(x, F_{1}(\varepsilon), e *\right)\left(z_{1}\right),\right.\right.\right. \\
& \mathscr{F}\left(z_{1}, F_{2}(\varepsilon), e *\right)\left(z_{2}\right), \ldots, \\
& \left.\left.\left.\mathscr{F}\left(z_{i-1}, F_{i}(\varepsilon), e *\right)(y)\right\}, \gamma\right\}, \delta\right\} \\
& \geq \sup _{z_{1}^{i} \in H} \min \left\{\mathscr{F}\left(z_{1}, F_{1}(\varepsilon), e *\right)(x),\right. \\
& \mathscr{F}\left(z_{2}, F_{2}(\varepsilon), e *\right)\left(z_{1}\right), \ldots, \\
& \left.\mathscr{F}\left(y, F_{i}(\varepsilon), e *\right)\left(z_{i-1}\right), \delta\right\} \\
& =\min \left\{\mathscr{F}\left(y, F_{i}(\varepsilon), F_{i-1}(\varepsilon), \ldots, F_{1}(\varepsilon), e *\right)(x), \delta\right\} \\
& =\min \left\{\mathscr{F}\left(y, F_{1}^{i}(\varepsilon), e *\right)(x), \delta\right\} .
\end{aligned}
$$

This implies $\max \left\{\min \left\{\mathscr{F}\left(x, F_{1}^{i}(\varepsilon), e *\right)(y), \delta\right\}, \gamma\right\} \quad \geq$ $\max \left\{\min \left\{\mathscr{F}\left(y, F_{1}^{i}(\varepsilon), e *\right)(x), \delta\right\}, \gamma\right\}$. In a similar way, we have $\max \left\{\min \left\{\mathscr{F}\left(y, F_{1}^{i}(\varepsilon), e *\right)(x), \delta\right\}, \gamma\right\} \geq \max \left\{\min \left\{\mathscr{F}\left(x, F_{1}^{i}(\varepsilon)\right.\right.\right.$, $e *)(y), \delta\}, \gamma\}$. Hence $\max \left\{\min \left\{\mathscr{F}\left(x, F_{1}^{i}(\varepsilon), e *\right)(y), \delta\right\}, \gamma\right\}=$ $\max \left\{\min \left\{\mathscr{F}_{1}^{i}(y, F(\varepsilon), e *)(x), \delta\right\}, \gamma\right\}$.

(2) Let $x, y, y_{1}^{n-1} \in H$ be such that $y \in f\left(x, y_{1}^{n-1}\right)$ and $\varepsilon \in A$. Then $\mathscr{F}(x, \underbrace{F(\varepsilon), \ldots, F(\varepsilon)}_{n-1})(y) \geq \min \left\{F(\varepsilon)_{y_{1}}^{y_{n-1}}\right\}$. Thus, we have

$$
\begin{aligned}
& \max \{F(\varepsilon)(x), \gamma\} \\
& \geq \max \{\min \{\mathscr{F}(\underbrace{F(\varepsilon), \ldots, F(\varepsilon)}_{n})(x), \delta\}, \gamma\} \\
& \geq \max \{\min \{F(\varepsilon)(y), \mathscr{F}(y, \underbrace{F(\varepsilon), \ldots, F(\varepsilon)}_{n-1})(x),
\end{aligned}
$$

$$
\begin{gathered}
\delta\}, \gamma\} \\
\geq \min \{F(\varepsilon)(y), \max \{\mathscr{F}(y, \underbrace{F(\varepsilon), \ldots, F(\varepsilon)}_{n-1})(x), \\
\gamma\}, \delta\} \\
\geq \min \{F(\varepsilon)(y), \mathscr{F}(x, \underbrace{F(\varepsilon), \ldots, F(\varepsilon)}_{n-1})(y), \delta\} \\
\geq \min \left\{F(\varepsilon)(y), F(\varepsilon)_{y_{1}}^{y_{n-1}}, \delta\right\} .
\end{gathered}
$$

This completes the proof.

Theorem 26. Let $\left\langle F_{k}, A\right\rangle$ be $\left(\epsilon_{\gamma}, \epsilon_{\gamma} \vee q_{\delta}\right)$-fuzzy soft invertible n-ary subhypergroup over $H$ for all $1 \leq k \leq n$ and $2 \leq$ $i \leq n$. Then $\mathfrak{F}(\left\langle F_{1}, A\right\rangle, \ldots,\left\langle F_{i}, A\right\rangle, \underbrace{\left\langle\widetilde{1_{e}}, A\right\rangle, \ldots,\left\langle\widetilde{1_{e}}, A\right\rangle}_{n-i})$ is an $\left(\epsilon_{\gamma}, \epsilon_{\gamma} \vee q_{\delta}\right)$-fuzzy soft invertible n-ary subhypergroup over $H$.

Proof. Since each $\left\langle F_{k}, A\right\rangle$ is an $\left(\epsilon_{\gamma}, \epsilon_{\gamma} \vee q_{\delta}\right)$-fuzzy soft invertible $n$-ary subhypergroup over $H$ for all $1 \leq k \leq n$, let $\varepsilon \in A$. It is clear that $\max \left\{F\left(F_{1}^{i}(\varepsilon), e *\right)(e), \gamma\right\} \geq \min \left\{\mathscr{F}\left(F_{1}^{i}(\varepsilon), e *\right)(x), \delta\right\}$ for all $x \in H$. From Lemma 23, for any $2 \leq j \leq n$, we have

$$
\begin{gathered}
\mathscr{F}(\underbrace{\mathscr{F}\left(F_{1}^{i}(\varepsilon), e *\right), \ldots, \mathscr{F}\left(F_{1}^{i}(\varepsilon), e *\right)}_{j}, e *) \\
=\mathscr{F}\left(\mathscr{F}(\underbrace{F_{1}(\varepsilon), \ldots, F_{1}(\varepsilon)}_{j}, e *), \ldots,\right. \\
\left.\mathscr{F}(\underbrace{F_{i}(\varepsilon), \ldots, F_{i}(\varepsilon)}_{j}, e *), e *\right) \\
{ }_{(\gamma, \delta)} \mathscr{F}\left(F_{1}^{i}(\varepsilon), e *\right) .
\end{gathered}
$$

In particular, we have

$$
\begin{aligned}
& \mathscr{F}(\underbrace{\mathscr{F}\left(F_{1}^{i}(\varepsilon), e *\right), \ldots, \mathscr{F}\left(F_{1}^{i}(\varepsilon), e *\right)}_{n}) \\
& { }_{(\gamma, \delta)} \mathscr{F}\left(F_{1}^{i}(\varepsilon), e *\right) .
\end{aligned}
$$

Now we show that 


$$
\begin{aligned}
\max & \left\{\min \left\{\mathscr{F}(x, \underbrace{\mathscr{F}\left(F_{1}^{i}(\varepsilon), e *\right), \ldots, \mathscr{F}\left(F_{1}^{i}(\varepsilon), e *\right)}_{n-1})(y), \delta\right\}, \gamma\right\} \\
& =\max \left\{\min \left\{\mathscr{F}(y, \underbrace{\mathscr{F}\left(F_{1}^{i}(\varepsilon), e *\right), \ldots, \mathscr{F}\left(F_{1}^{i}(\varepsilon), e *\right)}_{n-1})(x), \delta\right\}, \gamma\right\}
\end{aligned}
$$

for all $x, y \in H$; that is, $\mathscr{F}\left(F_{1}^{i}(\varepsilon), e *\right)$ is invertible. In fact, by Lemmas 23 and 25(1), we have

$$
\begin{aligned}
\max & \left\{\min \left\{\mathscr{F}(x, \underbrace{\mathscr{F}\left(F_{1}^{i}(\varepsilon), e *\right), \ldots, \mathscr{F}\left(F_{1}^{i}(\varepsilon), e *\right)}_{n-1})(y), \delta\right\}, \gamma\right\} \\
& =\max \left\{\min \left\{\mathscr{F}\left(x, F_{1}^{i}(\varepsilon), e *\right)(y), \delta\right\}, \gamma\right\} \\
& =\max \left\{\min \left\{\mathscr{F}\left(y, F_{1}^{i}(\varepsilon), e *\right)(x), \delta\right\}, \gamma\right\}=\max \left\{\min \left\{\mathscr{F}(y, \underbrace{\mathscr{F}\left(F_{1}^{i}(\varepsilon), e *\right), \ldots, \mathscr{F}\left(F_{1}^{i}(\varepsilon), e *\right)}_{n-1})(x), \delta\right\}, \gamma\right\} .
\end{aligned}
$$

Next, let $x, y, y_{1}^{n-1} \in H$ be such that $y \in f\left(x, y_{1}^{n-1}\right)$. Then by Lemma 25(2), we have $\max \left\{\mathscr{F}\left(F_{1}^{i}(\varepsilon), e *\right)(x), \gamma\right\} \geq$ $\min \left\{\mathscr{F}\left(F_{1}^{i}(\varepsilon), e *\right)_{y_{1}}^{y_{n-1}}, \mathscr{F}\left(F_{1}^{i}(\varepsilon), e *\right)(y), \delta\right\}$

Summing up the above arguments,

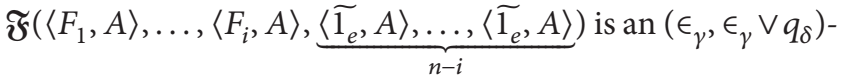
fuzzy soft invertible $n$-ary subhypergroup over $H$.

Theorem 27. Let $\langle F, A\rangle$ and $\langle G, B\rangle$ be two $\left(\epsilon_{\gamma}, \epsilon_{\gamma} \vee q_{\delta}\right)$ fuzzy soft invertible n-ary subhypergroups over $H$. Then both $\langle F, A\rangle \widetilde{\cap}\langle G, B\rangle$ and $\langle F, A\rangle \cap\langle G, B\rangle$ are $\left(\epsilon_{\gamma}, \epsilon_{\gamma} \vee q_{\delta}\right)$-fuzzy soft n-ary subhypergroups over $H$.

Proof. Let $\langle F, A\rangle$ and $\langle G, B\rangle$ be $\left(\epsilon_{\gamma}, \epsilon_{\gamma} \vee q_{\delta}\right)$-fuzzy soft invertible $n$-ary subhypergroups over $H$ and $\langle F, A\rangle \widetilde{\cap}\langle G, B\rangle=$ $\langle L, A \cup B\rangle$. Then, for any $\varepsilon \in A \cup B$, we consider the following cases.

Case $1(\varepsilon \in A-B)$. In this case, $L(\varepsilon)=F(\varepsilon)$. It follows that $L(\varepsilon)$ satisfies conditions (Fla), (F2a), and (F3a) since $\langle F, A\rangle$ is an $\left(\epsilon_{\gamma}, \epsilon_{\gamma} \vee q_{\delta}\right)$-fuzzy soft invertible $n$-ary subhypergroup over $S$.

Case $2(\varepsilon \in B-A)$. In this case, $L(\varepsilon)=G(\varepsilon)$. It follows that $L(\varepsilon)$ satisfies conditions (Fla), (F2a), and (F3a) since $\langle G, B\rangle$ is an $\left(\epsilon_{\gamma}, \epsilon_{\gamma} \vee q_{\delta}\right)$-fuzzy soft invertible $n$-ary subhypergroup over $S$.

Case $3(\varepsilon \in A \cap B)$. In this case, $L(\varepsilon)=F(\varepsilon) \cap G(\varepsilon)$. Now we show that $F(\varepsilon) \cap G(\varepsilon)$ satisfies conditions (Fla), (F2a), and (F3a).
(1) For any $x \in H$, we have

$$
\begin{aligned}
& \max \{(F(\varepsilon) \cap G(\varepsilon))(e), \gamma\} \\
& =\max \{\min \{F(\varepsilon)(e), G(\varepsilon)(e)\}, \gamma\} \\
& =\min \{\max \{F(\varepsilon)(e), \gamma\}, \max \{G(\varepsilon)(e), \gamma\}\} \\
& \geq \min \{F(\varepsilon)(x), G(\varepsilon)(x), \delta\} \\
& =\min \{(F(\varepsilon) \cap G(\varepsilon))(x), \delta\} .
\end{aligned}
$$

(2) Let $x, x_{1}^{n} \in H$ be such that $x \in f\left(x_{1}^{n}\right)$. Then

$$
\begin{aligned}
& \max \{(F(\varepsilon) \cap G(\varepsilon))(x), \gamma\} \\
& =\max \{\min \{F(\varepsilon)(x), G(\varepsilon)(x)\}, \gamma\} \\
& =\min \{\max \{F(\varepsilon)(x), \gamma\}, \max \{G(\varepsilon)(x), \gamma\}\} \\
& \geq \min \left\{F(\varepsilon)_{x_{1}}^{x_{n}}, G(\varepsilon)_{x_{1}}^{x_{n}}, \delta\right\} \\
& =\min \left\{(F(\varepsilon) \cap G(\varepsilon))_{x_{1}}^{x_{n}}, \delta\right\} .
\end{aligned}
$$

It follows that $\max \left\{\inf _{x \in f\left(x_{1}^{n}\right)}(F(\varepsilon) \cap G(\varepsilon))(x), \gamma\right\} \quad \geq$ $\min \left\{(F(\varepsilon) \cap G(\varepsilon))_{x_{1}}^{x_{n}}, \delta\right\}$. 
(3) Let $x, y, y_{1}^{n-1} \in H$ be such that $y \in f\left(x, y_{1}^{n-1}\right)$. Analogous to (2), we have

$$
\begin{aligned}
& \max \{(F(\varepsilon) \cap G(\varepsilon))(x), \gamma\} \\
& \quad \geq \min \left\{(F(\varepsilon) \cap G(\varepsilon))_{y_{1}}^{y_{n-1}},(F(\varepsilon) \cap G(\varepsilon))(y), \delta\right\} .
\end{aligned}
$$

Therefore, $\langle F, A\rangle \tilde{\cap}\langle G, B\rangle$ is an $\left(\epsilon_{\gamma}, \epsilon_{\gamma} \vee q_{\delta}\right)$-fuzzy soft $n$ ary subhypergroup over $H$. The case for $\langle F, A\rangle \cap\langle G, B\rangle$ can be similarly proved.

\section{The Homomorphism of $\left(\epsilon_{\gamma}, \epsilon_{\gamma} \vee q_{\delta}\right)$-Fuzzy Soft (Invertible) Subhypergroups}

In this section, we consider the homomorphism properties of $\left(\epsilon_{\gamma}, \epsilon_{\gamma} \vee q_{\delta}\right)$-fuzzy soft (invertible) subhypergroups. Let us first introduce the following definition.

Definition 28. Let $\mathscr{F} \mathcal{S}(U, E)$ and $\mathscr{F} \mathcal{S}\left(U^{\prime}, E^{\prime}\right)$ be two fuzzy soft classes, and let $\varphi: U \rightarrow U^{\prime}$ and $\phi: E \rightarrow E^{\prime}$ be mappings. Define a mapping $(\varphi, \phi): \mathscr{F} \mathcal{S}(U, E) \rightarrow$ $\mathscr{F} \mathcal{S}\left(U^{\prime}, E^{\prime}\right)$ by the following: for $\langle F, A\rangle \in \mathscr{F} \mathcal{S}(U, E)$, the image of $\langle F, A\rangle$ under $(\varphi, \phi)$, denoted by $(\varphi, \phi)\langle F, A\rangle=$ $\langle\varphi(F), \phi(A)\rangle$, is a fuzzy soft set in $\mathscr{F} \mathcal{S}\left(U^{\prime}, E^{\prime}\right)$ given by

$$
\begin{aligned}
& \varphi(F)\left(\varepsilon^{\prime}\right)\left(x^{\prime}\right) \\
& \quad= \begin{cases}\sup _{\varepsilon \in \phi^{-1}\left(\varepsilon^{\prime}\right) \cap A, x \in \varphi^{-1}\left(x^{\prime}\right)} F(\varepsilon)(x) & \text { if } \varphi^{-1}\left(x^{\prime}\right) \neq \emptyset, \\
0 & \text { otherwise, }\end{cases}
\end{aligned}
$$

for all $\varepsilon^{\prime} \in \phi(A)$ and $x^{\prime} \in U^{\prime}$. For $\left\langle F^{\prime}, A^{\prime}\right\rangle \in \mathscr{F} \mathcal{S}\left(U^{\prime}, E^{\prime}\right)$, the inverse image of $\left\langle F^{\prime}, A^{\prime}\right\rangle$ under $(\varphi, \phi)$, denoted by $(\varphi, \phi)^{-1}\left\langle F^{\prime}, A^{\prime}\right\rangle=\left\langle\varphi^{-1}\left(F^{\prime}\right), \phi^{-1}\left(A^{\prime}\right)\right\rangle$, is a fuzzy soft set in $\mathscr{F} \mathcal{S}(U, E)$ given by

$$
\varphi^{-1}\left(F^{\prime}\right)(\varepsilon)(x)=F^{\prime}(\phi(\varepsilon))(\varphi(x))
$$

for all $\varepsilon \in \phi^{-1}(B)$ and $x \in U$.

The following results can be easily deduced.
Lemma 29. Let $\langle F, A\rangle,\langle G, B\rangle \in \mathscr{F} \mathcal{S}(U, E)$ and $\varphi: U \rightarrow U^{\prime}$ and $\phi: E \rightarrow E^{\prime}$ be two mappings. Then

(1) $\langle F, A\rangle \Subset(\varphi, \phi)^{-1}((\varphi, \phi)\langle F, A\rangle)$ and $\langle F, A\rangle=$ $(\varphi, \phi)^{-1}((\varphi, \phi)\langle F, A\rangle)$ if both $\varphi$ and $\phi$ are injective;

(2) $(\varphi, \phi)(\langle F, A\rangle$ ก $\langle G, B\rangle) \Subset(\varphi, \phi)\langle F, A\rangle$ ก $(\varphi, \phi)\langle G, B\rangle$;

(3) $(\varphi, \phi)(\langle F, A\rangle \tilde{\cap}\langle G, B\rangle) \Subset(\varphi, \phi)\langle F, A\rangle \widetilde{\cap}(\varphi, \phi)\langle G, B\rangle$ if $\phi$ is injective;

(4) if $\langle F, A\rangle \Subset_{(\gamma, \delta)}\langle G, B\rangle$, then $\quad(\varphi, \phi)\langle F, A\rangle \Subset_{(\gamma, \delta)}(\varphi$, $\phi)\langle G, B\rangle$.

Lemma 30. Let $\left\langle F^{\prime}, A^{\prime}\right\rangle,\left\langle G^{\prime}, B^{\prime}\right\rangle \in \mathscr{F} \mathcal{S}\left(U^{\prime}, E^{\prime}\right)$ and $\varphi$ : $U \rightarrow U^{\prime}$ and $\phi: E \rightarrow E^{\prime}$ be two mappings. Then

(1) $(\varphi, \phi)\left((\varphi, \phi)^{-1}\left\langle F^{\prime}, A^{\prime}\right\rangle\right) \quad \Subset \quad\left\langle F^{\prime}, A^{\prime}\right\rangle$ and $(\varphi, \phi)\left((\varphi, \phi)^{-1}\left\langle F^{\prime}, A^{\prime}\right\rangle\right)=\left\langle F^{\prime}, A^{\prime}\right\rangle$ if both $\varphi$ and $\phi$ are surjective;

(2) $(\varphi, \phi)^{-1}\left(\left\langle F^{\prime}, A^{\prime}\right\rangle \cap\left\langle G^{\prime}, B^{\prime}\right\rangle\right)=(\varphi, \phi)^{-1}\left\langle F^{\prime}, A^{\prime}\right\rangle \pitchfork$ $(\varphi, \phi)^{-1}\left\langle G^{\prime}, B^{\prime}\right\rangle$

(3) $(\varphi, \phi)^{-1}\left(\left\langle F^{\prime}, A^{\prime}\right\rangle \widetilde{\cap}\left\langle G^{\prime}, B^{\prime}\right\rangle\right)=(\varphi, \phi)^{-1}\left\langle F^{\prime}, A^{\prime}\right\rangle \widetilde{\cap}$ $(\varphi, \phi)^{-1}\left\langle G^{\prime}, B^{\prime}\right\rangle$;

(4) if $\left\langle F^{\prime}, A^{\prime}\right\rangle \Subset_{(\gamma, \delta)}\left\langle G^{\prime}, B^{\prime}\right\rangle$, then $(\varphi, \phi)^{-1}\left\langle F^{\prime}, A^{\prime}\right\rangle \Subset_{(\gamma, \delta)}$ $(\varphi, \phi)^{-1}\left\langle G^{\prime}, B^{\prime}\right\rangle$.

Definition 31. Let $(H, f)$ and $\left(H^{\prime}, f^{\prime}\right)$ be two $n$-ary hypergroups with identities $e$ and $e^{\prime}$, respectively, and $\varphi$ a mapping from $H$ to $H^{\prime}$. Then, $\varphi$ is called a homomorphism if $\varphi(e)=e^{\prime}$ and $\varphi\left(f\left(x_{1}^{n}\right)\right)=f^{\prime}\left(\varphi\left(x_{1}\right), \ldots, \varphi\left(x_{n}\right)\right)$ for all $x_{1}^{n} \in H$. If such a homomorphism is surjective, injective, or bijective, it is called an epimorphism, a monomorphism, or an isomorphism.

Lemma 32. Let $(H, f)$ and $\left(H^{\prime}, f^{\prime}\right)$ be two $n$-ary hypergroups with identities $e$ and $e^{\prime}$, respectively, $\varphi: H \rightarrow H^{\prime} a$ homomorphism, and $\phi: E \rightarrow E^{\prime}$ a mapping. Then $(\varphi, \phi)\left(\mathfrak{F}\left(\langle F, A\rangle_{1}^{n}\right)\right) \Subset \mathfrak{F}^{\prime}\left((\varphi, \phi)\left\langle F_{1}, A_{1}\right\rangle, \ldots,(\varphi, \phi)\left\langle F_{n}, A_{n}\right\rangle\right)$ for all $\langle F, A\rangle_{1}^{n} \in \mathscr{F} \mathcal{S}(H, E)$ such that $\bigcap_{i=1}^{n} A_{i} \neq \emptyset$ and $(\varphi, \phi)\left(\mathfrak{F}\left(\langle F, A\rangle_{1}^{n}\right)\right)=\mathfrak{F}^{\prime}\left((\varphi, \phi)\left\langle F_{1}, A_{1}\right\rangle, \ldots,(\varphi, \phi)\left\langle F_{n}, A_{n}\right\rangle\right)$ if $\phi$ is injective.

Proof. Let $\langle F, A\rangle_{1}^{n} \in \mathscr{F} \mathcal{S}(H, E), \varepsilon^{\prime} \in \phi\left(\bigcap_{i=1}^{n} A_{i}\right) \subseteq$ $\bigcap_{i=1}^{n} \phi\left(A_{i}\right)$, and $x^{\prime} \in H^{\prime}$. If $\varphi^{-1}\left(x^{\prime}\right)=\emptyset$, then $\mathbb{F}^{\prime}\left(\varphi\left(F_{1}\right), \ldots, \varphi\left(F_{n}\right)\right)\left(\varepsilon^{\prime}\right)\left(x^{\prime}\right)=0=\varphi\left(\mathbb{F}\left(F_{1}^{n}\right)\left(\varepsilon^{\prime}\right)\right)\left(x^{\prime}\right)$. Otherwise, we have

$$
\begin{aligned}
\mathbb{F}^{\prime}( & \left.\left(F_{1}\right), \ldots, \varphi\left(F_{n}\right)\right)\left(\varepsilon^{\prime}\right)\left(x^{\prime}\right) \\
& =\mathscr{F}^{\prime}\left(\varphi\left(F_{1}\right)\left(\varepsilon^{\prime}\right), \ldots, \varphi\left(F_{n}\right)\left(\varepsilon^{\prime}\right)\right)\left(x^{\prime}\right) \\
& =\sup _{x^{\prime} \in f^{\prime}\left(x_{1}^{\prime}, \ldots, x_{n}^{\prime}\right)} \min \left\{\varphi\left(F_{1}\right)\left(\varepsilon^{\prime}\right)\left(x_{1}^{\prime}\right), \ldots, \varphi\left(F_{n}\right)\left(\varepsilon^{\prime}\right)\left(x_{n}^{\prime}\right)\right\} \\
& =\sup _{x_{1}^{\prime}, \ldots, x_{n}^{\prime} \in \operatorname{Im}(\varphi), x^{\prime} \in f^{\prime}\left(x_{1}^{\prime}, \ldots, x_{n}^{\prime}\right)} \min \left\{\sup _{\varepsilon_{1} \in \phi^{-1}\left(\varepsilon^{\prime}\right) \cap A_{1}, \varphi\left(x_{1}\right)=x_{1}^{\prime}} F_{1}\left(\varepsilon_{1}\right)\left(x_{1}\right), \ldots, \sup _{\varepsilon_{n} \in \phi^{-1}\left(\varepsilon^{\prime}\right) \cap A_{n}, \varphi\left(x_{n}\right)=x_{n}^{\prime}} F_{n}\left(\varepsilon_{n}\right)\left(x_{n}\right)\right\}
\end{aligned}
$$




$$
\begin{aligned}
& =\sup _{\substack{x_{1}^{\prime}, \ldots, x_{n}^{\prime} \in \operatorname{Im}(\varphi), x^{\prime} \in f^{\prime}\left(x_{1}^{\prime}, \ldots, x_{n}^{\prime}\right), \varepsilon_{1} \in \phi^{-1}\left(\varepsilon^{\prime}\right) \cap A_{1}, \ldots, \varepsilon_{n} \in \phi^{-1}\left(\varepsilon^{\prime}\right) \cap A_{n}}} \min \left\{F_{1}\left(\varepsilon_{1}\right)\left(x_{1}\right), \ldots, F_{n}\left(\varepsilon_{n}\right)\left(x_{n}\right)\right\} \\
& \geq \sup _{x^{\prime} \in f^{\prime}\left(\varphi\left(x_{1}\right), \ldots, \varphi\left(x_{n}\right)\right), \varepsilon \in \phi^{-1}\left(\varepsilon^{\prime}\right) \cap \cap_{i=1}^{n} A_{i}} \min \left\{F_{1}(\varepsilon)\left(x_{1}\right), \ldots, F_{n}(\varepsilon)\left(x_{n}\right)\right\} \\
& =\sup _{x^{\prime} \in \varphi\left(f\left(x_{1}, \ldots, x_{n}\right)\right), \varepsilon \in \phi^{-1}\left(\varepsilon^{\prime}\right) \cap \cap_{i=1}^{n} A_{i}} \min \left\{F_{1}(\varepsilon)\left(x_{1}\right), \ldots, F_{n}(\varepsilon)\left(x_{n}\right)\right\} \\
& =\sup _{x \in \varphi^{-1}\left(x^{\prime}\right), x \in f\left(x_{1}, \ldots, x_{n}\right), \varepsilon \in \phi^{-1}\left(\varepsilon^{\prime}\right) \cap n_{i=1}^{n} A_{i}} \min \left\{F_{1}(\varepsilon)\left(x_{1}\right), \ldots, F_{n}(\varepsilon)\left(x_{n}\right)\right\} \\
& =\sup _{x \in \varphi^{-1}\left(x^{\prime}\right), \varepsilon \in \phi^{-1}\left(\varepsilon^{\prime}\right) \cap n_{i=1}^{n} A_{i}} \mathscr{F}\left(F_{1}^{n}(\varepsilon)\right)(x)=\sup _{x \in \varphi^{-1}\left(x^{\prime}\right), \varepsilon \in \phi^{-1}\left(\varepsilon^{\prime}\right) \cap n_{i=1}^{n} A_{i}} \mathbb{F}\left(F_{1}^{n}\right)(\varepsilon)(x)=\varphi\left(\mathbb{F}\left(F_{1}^{n}\right)\left(\varepsilon^{\prime}\right)\right)\left(x^{\prime}\right) .
\end{aligned}
$$

Hence $(\varphi, \phi)\left(\mathfrak{F}\left(\langle F, A\rangle_{1}^{n}\right)\right) \quad \Subset \quad \mathfrak{F}^{\prime}\left((\varphi, \phi)\left\langle F_{1}, A_{1}\right\rangle, \ldots\right.$, $\left.(\varphi, \phi)\left\langle F_{n}, A_{n}\right\rangle\right)$. If $\phi$ is injective, then the symbol " $\geq$ " in the above proof can be replaced with "="; hence $(\varphi, \phi)\left(\mathfrak{F}\left(\langle F, A\rangle_{1}^{n}\right)\right)=\mathfrak{F}^{\prime}\left((\varphi, \phi)\left\langle F_{1}, A_{1}\right\rangle, \ldots,(\varphi, \phi)\left\langle F_{n}, A_{n}\right\rangle\right)$.

Theorem 33. Let $(H, f)$ and $\left(H^{\prime}, f^{\prime}\right)$ be two commutative $n$ ary hypergroups with identities e and $e^{\prime}$, respectively, $\varphi: H \rightarrow$ $H^{\prime}$ a homomorphism, and $\phi: E \rightarrow E^{\prime}$ an injective mapping. Let $\langle F, A\rangle$ be an $\left(\epsilon_{\gamma}, \epsilon_{\gamma} \vee q_{\delta}\right)$-fuzzy n-ary soft subhypergroup over $H$ which has the sup-property. Then, $(\varphi, \phi)\langle F, A\rangle$ is an $\left(\epsilon_{\gamma}, \epsilon_{\gamma} \vee q_{\delta}\right)$-fuzzy n-ary subhypergroup over $H^{\prime}$. If $\varphi$ is an epimorphism from $H$ onto $H^{\prime}$ and $\langle F, A\rangle$ is invertible, then $(\varphi, \phi)\langle F, A\rangle$ is also invertible.

Proof. Assume that $\langle F, A\rangle$ is an $\left(\epsilon_{\gamma}, \epsilon_{\gamma} \vee q_{\delta}\right)$-fuzzy $n$-ary soft subhypergroup over $H$ which has the sup-property and $\varepsilon^{\prime} \epsilon$ $\phi(A)$. Then there exists a unique $\varepsilon \in A$ such that $\phi(\varepsilon)=\varepsilon^{\prime}$ and we have the following.

(1) It is clear that $\max \left\{\varphi(F)\left(\varepsilon^{\prime}\right)\left(e^{\prime}\right), \gamma\right\} \quad \geq$ $\min \left\{\varphi(F)\left(\varepsilon^{\prime}\right)\left(x^{\prime}\right), \delta\right\}$ for all $x^{\prime} \in H$.

(2) By Lemma 32, we have

$$
\begin{aligned}
& \mathscr{F}^{\prime}(\underbrace{\varphi(F)\left(\varepsilon^{\prime}\right), \ldots, \varphi(F)\left(\varepsilon^{\prime}\right)}_{n}) \\
& =\varphi(\mathbb{F}(\underbrace{F, \ldots, F}_{n}))\left(\varepsilon^{\prime}\right) \subseteq \vee q_{(\gamma, \delta)} \varphi(F)\left(\varepsilon^{\prime}\right) .
\end{aligned}
$$

(3) Let $y^{\prime}, y_{1}^{\prime}, \ldots, y_{n-1}^{\prime} \in H^{\prime}$. If $\varphi^{-1}\left(y_{j}^{\prime}\right)=\emptyset(1 \leq j \leq n-1)$ or $\varphi^{-1}\left(y^{\prime}\right)=\emptyset$, then

$$
\begin{aligned}
& \max \left\{\varphi(F)\left(\varepsilon^{\prime}\right)\left(x^{\prime}\right), \gamma\right\} \geq 0 \\
& =\min \left\{\varphi(F)\left(\varepsilon^{\prime}\right)_{y_{1}^{\prime}}^{y_{n-1}^{\prime}}, \varphi(F)\left(\varepsilon^{\prime}\right)\left(y^{\prime}\right), \delta\right\}
\end{aligned}
$$

for $y^{\prime} \in f^{\prime}\left(x^{\prime}, y_{1}^{\prime}, \ldots, y_{n-1}^{\prime}\right)$. Otherwise, there exist $y, y_{1}^{n-1} \in$ $H$ such that $\varphi(y)=y^{\prime}, \varphi\left(y_{i}\right)=y_{i}^{\prime}, \varphi(F)\left(\varepsilon^{\prime}\right)\left(y^{\prime}\right)=F(\varepsilon)(y)$, and $\varphi(F)\left(\varepsilon^{\prime}\right)\left(y_{i}^{\prime}\right)=F(\varepsilon)\left(y_{i}\right)$ for all $1 \leq i \leq n-1$ since $\langle F, A\rangle$ has the sup-property. Now, for $y, y_{1}^{n-1} \in H$, since $\langle F, A\rangle$ is an $\left(\epsilon_{\gamma}, \epsilon_{\gamma} \vee q_{\delta}\right)$-fuzzy soft $n$-ary subhypergroup over $H$, there exists $x \in H$ such that $y \in f\left(x, y_{1}^{n-1}\right)$ and $\max \{F(\varepsilon)(x), \gamma\} \geq$ $\min \left\{F(\varepsilon)_{y_{1}}^{y_{n-1}}, F(\varepsilon)(y), \delta\right\}$. Hence we have

$$
\begin{aligned}
y^{\prime} & =\varphi(y) \in \varphi\left(f\left(x, y_{1}^{n-1}\right)\right) \\
& =f^{\prime}\left(\varphi(x), \varphi\left(y_{1}\right), \ldots, \varphi\left(y_{n-1}\right)\right) \\
& =f^{\prime}\left(\varphi(x), y_{1}^{\prime}, \ldots, y_{n-1}^{\prime}\right), \\
& \max \left\{\varphi(F)\left(\varepsilon^{\prime}\right)(\varphi(x)), \gamma\right\} \\
& =\max \left\{\sup _{\varphi(a)=\varphi(x)} F(\varepsilon)(a), \gamma\right\} \geq \max \{F(\varepsilon)(x), \gamma\} \\
& \geq \min \left\{F(\varepsilon)_{y_{1}}^{y_{n-1}}, F(\varepsilon)(y), \delta\right\} \\
& =\min \left\{\varphi(F)\left(\varepsilon^{\prime}\right)_{y_{1}^{\prime}}^{y_{n-1}^{\prime}}, \varphi(F)\left(\varepsilon^{\prime}\right)\left(y^{\prime}\right), \delta\right\} .
\end{aligned}
$$

Summing up the above analysis, $(\varphi, \phi)(\langle F, A\rangle)$ is an $\left(\epsilon_{\gamma}, \epsilon_{\gamma} \vee\right.$ $q_{\delta}$ )-fuzzy soft $n$-ary subhypergroup over $H^{\prime}$.

Next assume that $\varphi$ is an epimorphism from $H$ onto $H^{\prime}$ and that $\langle F, A\rangle$ is an invertible $\left(\epsilon_{\gamma}, \epsilon_{\gamma} \vee q_{\delta}\right)$-fuzzy soft $n$-ary subhypergroup over $H$. Then, for any $x^{\prime}, y^{\prime} \in H^{\prime}$, we have 


$$
\begin{aligned}
& \max \left\{\min \left\{\mathscr{F}^{\prime}(x^{\prime}, \underbrace{\varphi(F)\left(\varepsilon^{\prime}\right), \ldots, \varphi(F)\left(\varepsilon^{\prime}\right)}_{n-1})\left(y^{\prime}\right), \delta\right\}, \gamma\right\} \\
& =\max \left\{\min \left\{\sup _{\varphi(x)=x^{\prime}} \mathscr{F}^{\prime}(\varphi(x), \underbrace{\varphi(F)\left(\varepsilon^{\prime}\right), \ldots, \varphi(F)\left(\varepsilon^{\prime}\right)}_{n-1})\left(y^{\prime}\right), \delta\right\}, \gamma\right\} \\
& =\max \left\{\min \left\{\sup _{\varphi(x)=x^{\prime}} \varphi\left(\mathbb{F}(\widetilde{1_{x}}, \underbrace{F, \ldots, F}_{n-1})\right)\left(\varepsilon^{\prime}\right)\left(y^{\prime}\right), \delta\right\}, \gamma\right\} \\
& =\max \left\{\min \left\{\sup _{\varphi(x)=x^{\prime}, \varphi(y)=y^{\prime}} \mathscr{F}(x, \underbrace{F(\varepsilon), \ldots, F(\varepsilon)}_{n-1})(y), \delta\right\}, \gamma\right\} \\
& \left.=\sup _{\varphi(x)=x^{\prime}, \varphi(y)=y^{\prime}} \max _{\min }\{\mathscr{F}(x, \underbrace{F(\varepsilon), \ldots, F(\varepsilon)}_{n-1})(y), \delta\}, \gamma\right\} .
\end{aligned}
$$

Similarly, we have

$$
\begin{aligned}
\max & \left\{\min \left\{\mathscr{F}^{\prime}(y^{\prime}, \underbrace{\varphi(F)\left(\varepsilon^{\prime}\right), \ldots, \varphi(F)\left(\varepsilon^{\prime}\right)}_{n-1})\left(x^{\prime}\right), \delta\right\}, \gamma\right\} \\
& =\sup _{\varphi(x)=x^{\prime}, \varphi(y)=y^{\prime}} \max \{\min \{\mathscr{F}(y, \underbrace{F(\varepsilon), \ldots, F(\varepsilon)}_{n-1})(x), \delta\}, \gamma\} .
\end{aligned}
$$

It follows that

$$
\begin{aligned}
\max & \left\{\min \left\{\mathscr{F}^{\prime}(x^{\prime}, \underbrace{\varphi(F)\left(\varepsilon^{\prime}\right), \ldots, \varphi(F)\left(\varepsilon^{\prime}\right)}_{n-1})\left(y^{\prime}\right), \delta\right\}, \gamma\right\} \\
& =\max \left\{\min \left\{\mathscr{F}^{\prime}(y^{\prime}, \underbrace{\varphi(F)\left(\varepsilon^{\prime}\right), \ldots, \varphi(F)\left(\varepsilon^{\prime}\right)}_{n-1})\left(x^{\prime}\right), \delta\right\}, \gamma\right\}
\end{aligned}
$$

since $\max \{\min \{\mathscr{F}(x, \underbrace{F(\varepsilon), \ldots, F(\varepsilon)}_{n-1})(y), \delta\}, \gamma\}$ $\max \{\min \{\mathscr{F}(y, \underbrace{F(\varepsilon), \ldots, F(\varepsilon)}_{n-1})(x), \delta\}, \gamma\}$.

Therefore, $(\varphi, \phi)\langle F, A\rangle$ is invertible.

Theorem 34. Let $(H, f)$ and $\left(H^{\prime}, f^{\prime}\right)$ be two commutative $n$-ary hypergroups with identities $e$ and $e^{\prime}$, respectively, $\varphi$ : $H \rightarrow H^{\prime}$ a homomorphism, and $\phi: E \rightarrow E^{\prime}$ a mapping. Let $\left\langle F^{\prime}, A^{\prime}\right\rangle$ be an $\left(\epsilon_{\gamma}, \epsilon_{\gamma} \vee q_{\delta}\right)$-fuzzy soft n-ary subhypergroup over $H^{\prime}$. Then $(\varphi, \phi)^{-1}\left\langle F^{\prime}, A^{\prime}\right\rangle$ is an $\left(\epsilon_{\gamma}, \epsilon_{\gamma} \vee q_{\delta}\right)$-fuzzy soft n-ary subhypergroup over $H$. If both $\varphi$ and $\phi$ are injective $=\quad$ and $\left\langle F^{\prime}, A^{\prime}\right\rangle$ is an $\left(\epsilon_{\gamma}, \epsilon_{\gamma} \vee q_{\delta}\right)$-fuzzy soft (invertible) n-ary subhypergroup over $H^{\prime}$, then $(\varphi, \phi)^{-1}\left\langle F^{\prime}, A^{\prime}\right\rangle$ is an $\left(\epsilon_{\gamma}, \epsilon_{\gamma} \vee\right.$ $q_{\delta}$ )-fuzzy soft (invertible) $n$-ary subhypergroup over $H$.

Proof. Assume that $\left\langle F^{\prime}, A^{\prime}\right\rangle$ is an $\left(\epsilon_{\gamma}, \epsilon_{\gamma} \vee q_{\delta}\right)$-fuzzy soft $n$ ary subhypergroup over $H^{\prime}$. Let $\varepsilon \in \phi^{-1}\left(A^{\prime}\right)$. Then we have the following.

(1) For any $x \in H, \max \left\{\varphi^{-1}\left(F^{\prime}\right)(\varepsilon)(e), \gamma\right\}=$ $\max \left\{F^{\prime}(\phi(\varepsilon))(\varphi(e)), \gamma\right\} \geq \min \left\{F^{\prime}(\phi(\varepsilon))(\varphi(x)), \delta\right\}=$ $\min \left\{\varphi^{-1}\left(F^{\prime}\right)(\varepsilon)(x), \delta\right\}$. 
(2) Let $x, x_{1}^{n} \in H$ be such that $x \in f\left(x_{1}^{n}\right)$. Then $\varphi(x) \in$ $\varphi\left(f\left(x_{1}^{n}\right)\right)=f^{\prime}\left(\varphi(x)_{1}^{n}\right)$ and so

$$
\begin{aligned}
& \max \left\{\varphi^{-1}\left(F^{\prime}\right)(\varepsilon)(x), \gamma\right\} \\
& =\max \left\{F^{\prime}(\phi(\varepsilon))(\varphi(x)), \gamma\right\} \\
& \geq \min \left\{F^{\prime}(\phi(\varepsilon))\left(\varphi\left(x_{1}\right)\right), \ldots, F^{\prime}(\phi(\varepsilon))\left(\varphi\left(x_{n}\right)\right), \delta\right\} \\
& =\min \left\{\varphi^{-1}\left(F^{\prime}\right)(\varepsilon)_{x_{1}}^{x_{n}}, \delta\right\} .
\end{aligned}
$$

It follows that $\max \left\{\inf _{x \in f\left(x_{1}^{n}\right)} \varphi^{-1}\left(F^{\prime}\right)(\varepsilon)(z), \gamma\right\} \quad \geq$ $\min \left\{\varphi^{-1}\left(F^{\prime}\right)(\varepsilon)_{x_{1}}^{x_{n}}, \delta\right\}$

(3) Let $x, y, y_{1}^{n-1} \in H$ be such that $y \in f\left(x, y_{1}^{n-1}\right)$. Then $\varphi(y) \in f^{\prime}\left(\varphi(x), \varphi\left(y_{1}\right), \ldots, \varphi\left(y_{n-1}\right)\right)$. Since $\left\langle F^{\prime}, A^{\prime}\right\rangle$ is invertible, by Lemma 25(2), we have

$$
\begin{aligned}
& \max \left\{\varphi^{-1}\left(F^{\prime}\right)(\varepsilon)(x), \gamma\right\} \\
& =\max \left\{F^{\prime}(\phi(\varepsilon))(\varphi(x)), \gamma\right\} \\
& \geq \min \left\{F^{\prime}(\phi(\varepsilon))\left(\varphi\left(y_{1}\right)\right), \ldots, F^{\prime}(\phi(\varepsilon))\left(\varphi\left(y_{n-1}\right)\right),\right. \\
& \left.F^{\prime}(\phi(\varepsilon))(\varphi(y)), \delta\right\} \\
& =\min \left\{\varphi^{-1}\left(F^{\prime}\right)(\varepsilon)_{y_{1}}^{y_{n-1}}, \varphi^{-1}\left(F^{\prime}\right)(\varepsilon)(y), \delta\right\} .
\end{aligned}
$$

Summing up the above analysis, $(\varphi, \phi)^{-1}\left\langle F^{\prime}, A^{\prime}\right\rangle$ is an $\left(\epsilon_{\gamma}, \epsilon_{\gamma} \vee q_{\delta}\right)$-fuzzy $n$-ary soft subhypergroup over $H$. If both $\varphi$ and $\phi$ are injective and $\left\langle F^{\prime}, A^{\prime}\right\rangle$ is an $\left(\epsilon_{\gamma}, \epsilon_{\gamma} \vee q_{\delta}\right)$-fuzzy soft invertible $n$-ary subhypergroup over $H^{\prime}$, it is easy to check that $(\varphi, \phi)^{-1}\left\langle F^{\prime}, A^{\prime}\right\rangle$ is an $\left(\epsilon_{\gamma}, \epsilon_{\gamma} \vee q_{\delta}\right)$-fuzzy soft invertible $n$-ary subhypergroup over $H$.

\section{Conclusions}

In this paper, our aim is to promote the research and development of fuzzy soft technology by studying fuzzy soft $n$-ary hypergroups which generalize some algebra structures: soft groups, fuzzy $n$-ary hypergroups, fuzzy hypergroups, and so on. The goal is to explain new methodological development in $n$-ary hypergroups which will also be of growing importance in the future. The results presented in this paper can hopefully provide more insight into and a full understanding of fuzzy soft set theory and algebraic hyperstructures. Our future work on this topic will focus on studying some other classes of fuzzy soft $n$-ary hypergroups.

\section{Conflict of Interests}

The authors declare that there is no conflict of interests regarding the publication of this paper.

\section{Acknowledgment}

This research was supported by the TianYuan Special Funds of the National Natural Science Foundation of China (11226264).

\section{References}

[1] D. Molodtsov, "Soft set theory-first results," Computers and Mathematics with Applications, vol. 37, no. 4-5, pp. 19-31, 1999.

[2] F. Feng, Y. B. Jun, X. Liu, and L. Li, "An adjustable approach to fuzzy soft set based decision making," Journal of Computational and Applied Mathematics, vol. 234, no. 1, pp. 10-20, 2010.

[3] N. Çağman and S. Enginoğlu, "Soft matrix theory and its decision making," Computers and Mathematics with Applications, vol. 59, no. 10, pp. 3308-3314, 2010.

[4] N. Çağman and S. Enginoğlu, "Soft set theory and uni-int decision making," European Journal of Operational Research, vol. 207, no. 2, pp. 848-855, 2010.

[5] P. K. Maji, A. R. Roy, and R. Biswas, "An application of soft sets in a decision making problem," Computers and Mathematics with Applications, vol. 44, no. 8-9, pp. 1077-1083, 2002.

[6] A. R. Roy and P. K. Maji, "A fuzzy soft set theoretic approach to decision making problems," Journal of Computational and Applied Mathematics, vol. 203, no. 2, pp. 412-418, 2007.

[7] D. Chen, E. C. C. Tsang, D. S. Yeung, and X. Wang, "The parameterization reduction of soft sets and its applications," Computers and Mathematics with Applications, vol. 49, no. 5-6, pp. 757-763, 2005.

[8] Y. Zou and Z. Xiao, "Data analysis approaches of soft sets under incomplete information," Knowledge-Based Systems, vol. 21, no. 8, pp. 941-945, 2008.

[9] Z. Xiao, K. Gong, and Y. Zou, "A combined forecasting approach based on fuzzy soft sets," Journal of Computational and Applied Mathematics, vol. 228, no. 1, pp. 326-333, 2009.

[10] P. K. Maji, R. Biswas, and A. R. Roy, "Soft set theory," Computers and Mathematics with Applications, vol. 45, no. 4-5, pp. 555-562, 2003.

[11] M. I. Ali, F. Feng, X. Liu, W. K. Min, and M. Shabir, "On some new operations in soft set theory," Computers and Mathematics with Applications, vol. 57, no. 9, pp. 1547-1553, 2009.

[12] K. Qin and Z. Hong, "On soft equality," Journal of Computational and Applied Mathematics, vol. 234, no. 5, pp. 1347-1355, 2010.

[13] P. K. Maji, R. Biswas, and A. R. Roy, "Fuzzy soft sets," Journal of Fuzzy Mathematics, vol. 9, no. 3, pp. 589-602, 2001.

[14] P. Majumdar and S. K. Samanta, "Generalised fuzzy soft sets," Computers and Mathematics with Applications, vol. 59, no. 4, pp. 1425-1432, 2010.

[15] P. K. Maji, R. Biswas, and A. R. Roy, "Intuitionistic fuzzy soft sets," Journal of Fuzzy Mathematics, vol. 9, no. 3, pp. 677-692, 2001.

[16] P. K. Maji, A. R. Roy, and R. Biswas, "On intuitionistic fuzzy soft sets," Journal of Fuzzy Mathematics, vol. 12, no. 3, pp. 669-683, 2004.

[17] Y. Jiang, Y. Tang, and Q. Chen, "An adjustable approach to intuitionistic fuzzy soft sets based decision making," Applied Mathematical Modelling, vol. 35, no. 2, pp. 824-836, 2011.

[18] H. Aktaş and N. Çağman, "Soft sets and soft groups," Information Sciences, vol. 177, no. 13, pp. 2726-2735, 2007. 
[19] A. Aygünoğlu and H. Aygün, "Introduction to fuzzy soft groups," Computers and Mathematics with Applications, vol. 58, no. 6, pp. 1279-1286, 2009.

[20] F. Feng, Y. B. Jun, and X. Zhao, "Soft semirings," Computers and Mathematics with Applications, vol. 56, no. 10, pp. 2621-2628, 2008.

[21] Y. B. Jun, "Soft BCK/BCI-algebras," Computers and Mathematics with Applications, vol. 56, no. 5, pp. 1408-1413, 2008.

[22] Y. B. Jun and C. H. Park, "Applications of soft sets in ideal theory of BCK/BCI-algebras," Information Sciences, vol. 178, no. 11, pp. 2466-2475, 2008.

[23] Y. B. Jun, K. J. Lee, and C. H. Park, "Soft set theory applied to ideals in $d$-algebras," Computers and Mathematics with Applications, vol. 57, no. 3, pp. 367-378, 2009.

[24] U. Acar, F. Koyuncu, and B. Tanay, "Soft sets and soft rings," Computers and Mathematics with Applications, vol. 59, no. 11, pp. 3458-3463, 2010.

[25] J. Zhan and Y. B. Jun, "Soft BL-algebras based on fuzzy sets," Computers and Mathematics with Applications, vol. 59, no. 6, pp. 2037-2046, 2010.

[26] B. Davvaz and P. Corsini, "Fuzzy $n$-ary hypergroups," Journal of Intelligent and Fuzzy Systems, vol. 18, no. 4, pp. 377-382, 2007.

[27] B. Davvaz, P. Corsini, and V. Leoreanu-Fotea, "Fuzzy $n$-ary subpolygroups," Computers and Mathematics with Applications, vol. 57, no. 1, pp. 141-152, 2009.

[28] B. Dawaz and T. Vougiouklis, " $n$-ary hypergroups," Iranian Journal of Science and Technology, Transaction A: Science, vol. 30, no. 2, pp. 165-174, 2006.

[29] V. Leoreanu-Fotea and P. Corsini, "Isomorphisms of hypergroups and of $n$-hypergroups with applications," Soft Computing, vol. 13, no. 10, pp. 985-994, 2009.

[30] V. Leoreanu-Fotea and B. Davvaz, " $n$-hypergroups and binary relations," European Journal of Combinatorics, vol. 29, no. 5, pp. 1207-1218, 2008.

[31] V. Leoreanu-Fotea and B. Davvaz, "Roughness in $n$-ary hypergroups," Information Sciences, vol. 178, no. 21, pp. 4114-4124, 2008.

[32] S. Yamak, O. Kazanci, and B. Davvaz, "Applications of interval valued $t$-norms ( $t$-conorms) to fuzzy $n$-ary sub-hypergroups," Information Sciences, vol. 178, no. 20, pp. 3957-3972, 2008.

[33] Y. Yin, J. Zhan, D. Xu, and J. Wang, "The L-fuzzy hypermodules," Computers and Mathematics with Applications, vol. 59, no. 2, pp. 953-963, 2010.

[34] Y. Yin, J. Zhan, and P. Corsini, "Fuzzy roughness of $n$-ary hypergroups based on a complete residuated lattice," Neural Computing and Applications, vol. 20, no. 1, pp. 41-57, 2011.

[35] Y. Yin, B. Davvaz, and J. Zhan, "A fuzzy view of $\Gamma$-hyperrings," Neural Computing and Applications, vol. 21, no. 5, pp. 979-992, 2012.

[36] J. Zhan, B. Davvaz, and K. P. Shum, "A new view of fuzzy hypernear-rings," Information Sciences, vol. 178, no. 2, pp. 425438, 2008.

[37] J. Zhan, B. Davvaz, and K. P. Shum, "Generalized fuzzy hyperideals of hyperrings," Computers and Mathematics with Applications, vol. 56, no. 7, pp. 1732-1740, 2008.

[38] J. Zhan, B. Davvaz, and K. P. Shum, "On probabilistic n-ary hypergroups," Information Sciences, vol. 180, no. 7, pp. 1159-1166, 2010.

[39] P. M. Pu and Y. M. Liu, "Fuzzy topology I: neighbourhood structure of a fuzzy point and Moore-Smith convergence,"
Journal of Mathematical Analysis and Applications, vol. 76, pp. 571-599, 1980.

[40] Y. Yin and J. Zhan, "New types of fuzzy filters of BL-algebras," Computers and Mathematics with Applications, vol. 60, no. 7, pp. 2115-2125, 2010.

[41] X. Ma and J. Zhan, "On $(\epsilon, \in \mathrm{V} q)$-fuzzy filters of $B L$-algebras," Journal of Systems Science and Complexity, vol. 21, no. 1, pp. 144158, 2008.

[42] X. Ma, J. Zhan, and W. A. Dudek, "Some kinds of $(\bar{\epsilon}, \bar{\epsilon} \vee \bar{q})$ fuzzy filters of BL-algebras," Computers and Mathematics with Applications, vol. 58, no. 2, pp. 248-256, 2009.

[43] X. Ma, J. Zhan, and Y. B. Jun, "Some kinds of $\left(\epsilon_{\gamma}, \epsilon_{\gamma}, \vee q \delta\right)$ fuzzy ideals of BCI-algebras," Computers and Mathematics with Applications, vol. 61, no. 4, pp. 1005-1015, 2011.

[44] Y. Yin and J. Zhan, "The characterization of ordered semigroups in terms of fuzzy soft ideals," Bulletin of the Malaysian Mathematical Sciences Society, vol. 35, no. 4, pp. 997-1015, 2012.

[45] Y. Yin and J. Zhan, "The characterizations of hemirings in terms of fuzzy soft h-ideals," Neural Computing and Applications, vol. 21, pp. S43-S57, 2012.

[46] Y. Yin, Y. B. Jun, and Z. Yang, "General forms of $(\alpha, \beta)$-fuzzy ideals of ordered semigroups," Iranian Journal of Fuzzy Systems, vol. 9, pp. 99-113, 2012.

[47] L. A. Zadeh, "Fuzzy sets," Information and Control, vol. 8, no. 3, pp. 338-353, 1965. 


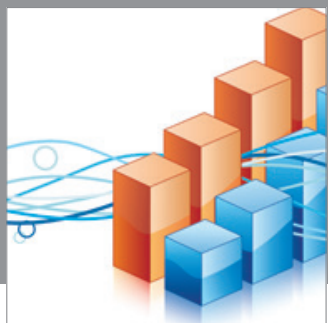

Advances in

Operations Research

mansans

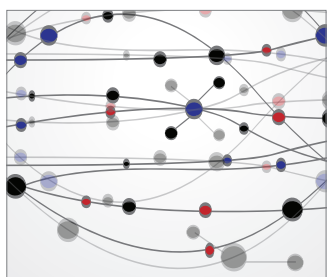

The Scientific World Journal
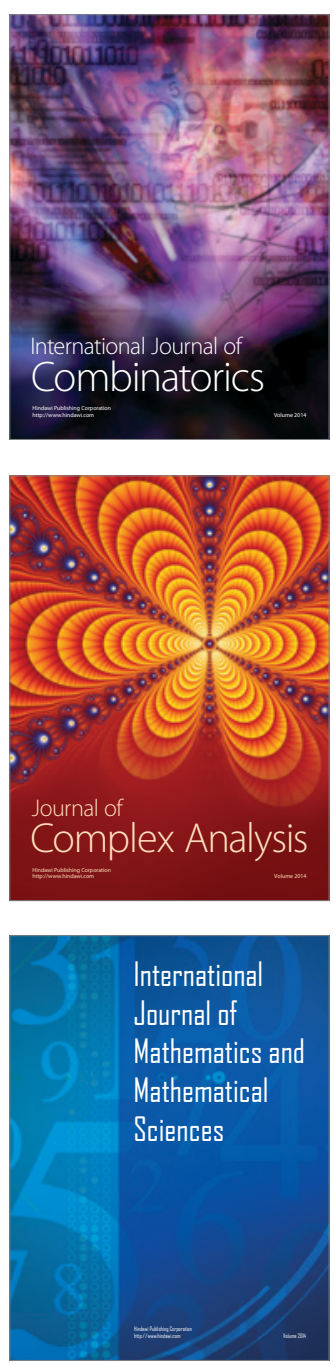
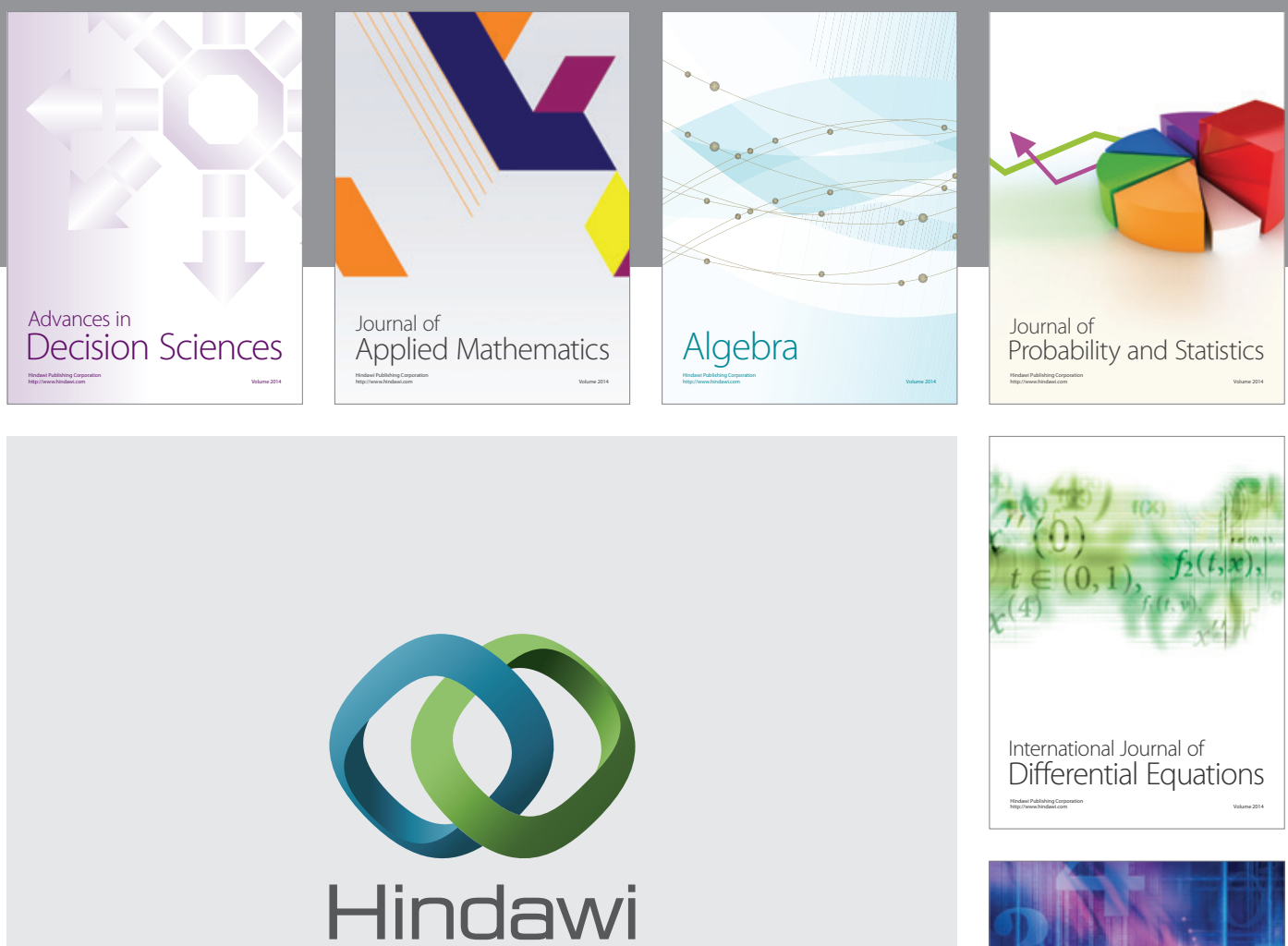

Submit your manuscripts at http://www.hindawi.com
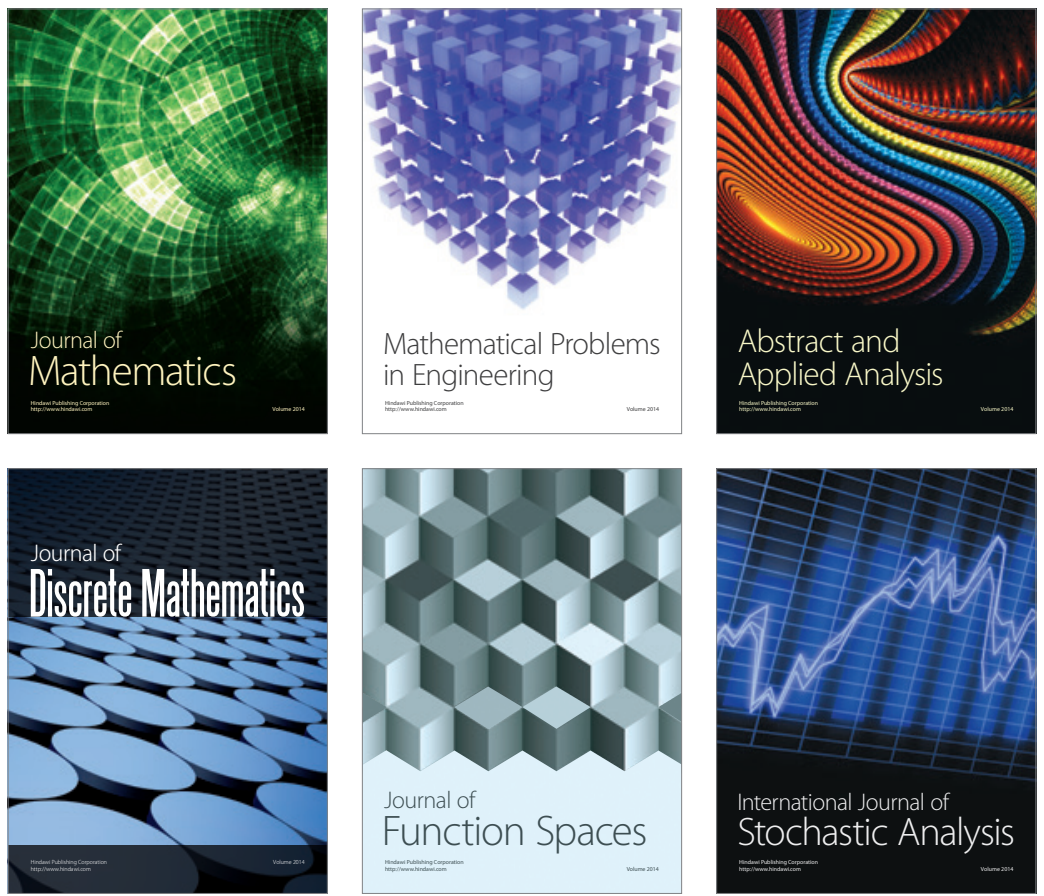

Journal of

Function Spaces

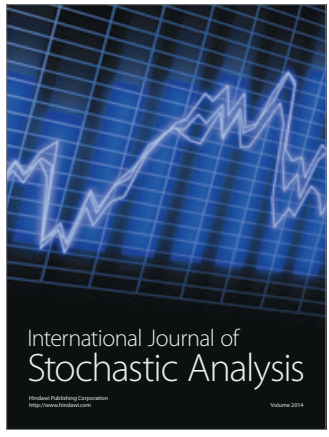

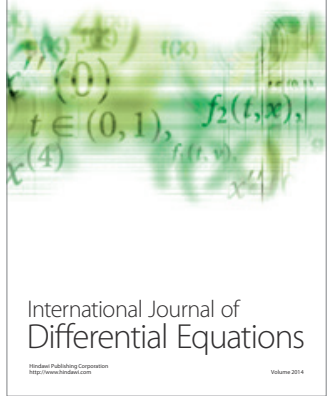
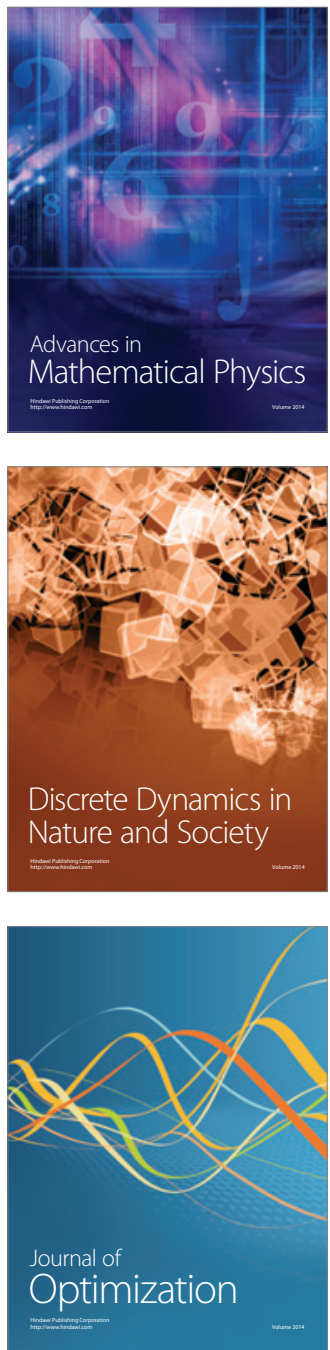